\title{
Mass loss evolution and the formation of detached shells around TP-AGB stars ${ }^{\star}$
}

\author{
L. Mattsson ${ }^{1}$, S. Höfner ${ }^{1}$, and F. Herwig ${ }^{2,3}$ \\ 1 Department of Astronomy and Space Physics, Uppsala University, Box 515, 75120 Uppsala, Sweden \\ e-mail: mattsson@astro.uu.se \\ 2 Keele Astrophysics Group, School of Physical and Geographical Sciences, Keele University, Stafford shire ST5 5BG, UK \\ e-mail: hoefner@astro.uu.se \\ 3 Theoretical Astrophysics Group, LANL, Los Alamos, NM 87545, USA \\ e-mail: fherwig@astro.keele.ac.uk
}

Received 8 September 2006 / Accepted 28 April 2007

ABSTRACT

\begin{abstract}
Context. The origin of the so called "detached shells" around AGB stars is not fully understood, but two common hypotheses state that these shells form either through the interaction of distinct wind phases or an eruptive mass loss associated with a He-shell flash. We present a model of the formation of detached shells around thermal pulse asymptotic giant branch (TP-AGB) stars, based on detailed modelling of mass loss and stellar evolution, leading to a combination of eruptive mass loss and wind interaction.

Aims. The purpose of this paper is first of all to connect stellar evolution with wind and mass loss evolution and demonstrate its consistency with observations, but also to show how thin detached shells around TP-AGB stars can be formed. Previous attempts to link mass loss evolution with the formation of detached shells were based on approximate prescriptions for the mass loss and have not included detailed modelling of the wind formation as we do here.

Methods. Using stellar parameters sampled from an evolutionary track for a $2 M_{\odot}$ star, we have computed the time evolution of the atmospheric layers and wind acceleration region during a typical thermal pulse with detailed radiation hydrodynamical models including dust formation. Based on these results, we simulate the subsequent circumstellar envelope (CSE) evolution using a spherical hydrodynamic model.

Results. We find that existing simple mass loss prescriptions all suggest different mass loss evolutions and that they differ from our detailed wind modelling. The most important factor for the formation of a detached shell is the wind velocity evolution which has a strong impact on the wind interaction and the resulting pile-up of matter. Our CSE model shows that a thin shell structure may be formed as a consequence of a rather short phase of intense mass loss in combination with a significant variation in the wind velocity, as obtained by our wind models. This situation can only be obtained for a limited range of amplitudes for the piston boundary used in the dynamic atmosphere models.

Conclusions. The combined mass loss eruption and wind interaction scenario for the formation of detached shells around AGB stars (suggested by previous work) is confirmed by the present modelling. Changes in mass loss rate and wind velocity due to a He-shell flash are adequate for creating distinct wind phases and a "snow plow effect" that is necessary to form a geometrically thin detached shell. The derived properties of the shell (i.e. radius, thickness and density) are more or less consistent with existing observational constraints.
\end{abstract}

Key words. stars: AGB and post-AGB - stars: atmospheres - stars: carbon - stars: circumstellar matter - stars: evolution stars: mass-loss

\section{Introduction}

The origin of the so called "detached shells" around TP-AGB stars in a late evolutionary stage is not fully understood. As a phenomenon, these structures have been known for about 20 years (see e.g. Olofsson et al. 1987). The idea that they were connected with He-shell flashes soon emerged, but the exact formation mechanism remained a matter of debate (Olofsson et al. 1990). In fact, it is still not clear that detached shells are only of internal origin. Wareing et al. (2006) have recently shown that shell-like structures like these can emerge from the interaction of an AGB wind with the interstellar medium (ISM) in which the star is moving. The shell structure could then be explained as a bow shock into the ISM.

^ Appendix A is only available in electronic form at http://www. aanda.org
A more common, older hypothesis for how these structures are formed, states that they arise from the interaction between a fast and a slow wind (two-wind interaction) from the central star. As the fast wind propagates outwards, it will sweep up material ejected through the slow wind and create a shell-like structure. This shell then contains matter ejected by the fast wind, together with swept up matter ejected by the slow wind. In order to work, this hypothesis needs a sufficiently high mass loss rate and wind velocity associated with the fast wind, as well as a non-negligable pre-flash wind (for more details, see Steffen et al. 1998; Olofsson et al. 2000; Schöier et al. 2005, and references therein). Kwok et al. (1978) presented an analytic wind interaction model in which a fast wind is running into a slower wind - each having a constant mass-loss rate and constant velocity. Hence, the distance from the central star to the interaction region is expected to increase linearly over time. This simple picture has been used to link observed mass loss 
properties with the presence of detached shells (Olofsson et al. 1993; Schöier et al. 2001, 2005).

Another early hypothesis (Zuckerman 1993) for the formation of thin shell structures suggests that a short event of high mass loss (with a non-evolving wind velocity), which we refer to as a "mass loss eruption", would simply translate into a correspondingly narrow, high-density shell moving through the circumstellar envelope. This, however, was shown to be an insufficient mechanism for the formation of detached shells (Steffen et al. 1997, 1998). But the same mass loss evolution could still produce the detached shell phenomenon if the wind before the flash is slower than the wind during the eruption, due to the sweep-up of matter that occurs as the matter released by the mass loss eruption propagates through the CSE created by the previous mass loss. This mass loss eruption scenario is most likely tied to a He-shell flash (thermal pulse) as suggested by Olofsson et al. (1990) and Vassiliadis \& Wood (1993). Under the assumption that the slow (pre-flash) stellar wind represents an ordinary state of mass loss for a TP-AGB star there is still the need of a period of relatively high mass loss and an enhanced outflow velocity, also in an interacting wind scenario.

Previous numerical models of the connection between a Heshell flash and the formation of a detached shell have provided some support for the wind-interaction hypothesis, but as mentioned above, only as a partial explanation. Steffen \& Schönberner (2000), from hereon abbrevated SS2000, confirmed this picture of a detached shell forming from a mass loss eruption and subsequent wind interaction qualitatively, using a spherical hydrodynamic model, altough the mass loss evolution is prescribed, not modelled, i.e. a more or less ad hoc assumption about the inner boundary.

A rather detailed 3D model of the hydrodynamical features of a detached shell was presented by Myasnikov et al. (2000), where the gasdynamic features of detached shells around a carbon rich TP-AGB star with variable mass loss rate were investigated numerically. They find that these shells are unstable to turbulence and that Rayleigh-Taylor instabilities develop in the radiative case. Furthermore they find that $2 \mathrm{D}$ calculations show quite different features compared with the $3 \mathrm{D}$ case and that the structures of the predicted shells are dependent on the initial basic wind properties. The latter is a very important finding. In order to confirm the two-wind interaction hypothesis on a more detailed level, it is thus cruical to connect the evolution of the stellar parameters $\left(M, L_{\star}, T_{\text {eff }}\right)$ with the mass loss and wind evolution during the He-flash event.

To understand the mass loss evolution of TP-AGB stars it is important to know how stellar evolution affects the physics of the atmosphere and consequently the formation of a stellar wind. This can be done by feeding the stellar parameters resulting from a stellar evolution model into a dynamic stellar atmosphere model. The models for the atmospheres and winds used in the present paper are computed using frequency-dependent radiative transfer for the gas and dust, including detailed microphysics of the dust grains and their formation (described in Höfner et al. 2003, and Andersen et al. 2003) in combination with time-dependent hydrodynamics. It has been shown that synthetic opacity sampling spectra for carbon-rich AGB stars based on these models reproduce the observed spectral energy distributions of these stars quite well (Gautschy-Loidl et al. 2004). Furthermore, for the first time, these models are also able to simultaneously reproduce the observed time-dependent behaviour of fundamental, first and second overtone rotation-vibration lines of $\mathrm{CO}$, which are formed in the outflow, wind acceleration region and atmosphere respectively (Nowotny et al. 2005a,b).
The mass loss properties, however, are more difficult to compare with observations. Derivations of "observed" rates of mass loss (e.g. Schöier et al. 2005) rely on stellar parameters and absolute distances that can be uncertain in many cases, which means that observations can only provide limited constraints on mass loss rates and dust-to-gas ratios. Modelling detached shells might therefore be an independent test of the mass loss properties of our dynamic atmosphere model.

In this paper we combine this radiation-hydrodynamics (RHD) model of a pulsating TP-AGB atmosphere and wind acceleration region with a detailed stellar evolution model and a simple spherical hydrodynamic model to follow the dynamical evolution of the CSE on long time scales and study the formation of a geometrically thin detached shell around the star. We are thus dealing with three different scales: the internal evolution, the atmosphere and the effects on the CSE. As this paper shows, our theoretically expected wind properties are such that a thin detached shell is formed as a natural consequence of a He-shell flash.

\section{Theory and method}

\subsection{General description}

On a descriptive level, the basic idea behind the modelling is to connect models of three different regimes (see Table 1). The starting point is a pre-computed model of the internal evolution of a star with a main sequence mass of $2 M_{\odot}$ and an initial metallicity of $Z=0.01$. This track is not chosen for any particular reason, other than that it is consistent with the type of carbon stars with known detached shells. The resulting stellar parameters $\left(M, L_{\star}, T_{\text {eff }}, \mathrm{C} / \mathrm{O}\right)$ are sampled with suitable time intervals around a thermal pulse. These parameter values are then fed into an RHD atmosphere model that provides us with the mass loss properties. Knowing these properties we can construct a time dependent inner boundary condition for a hydrodynamic model of the larger-scale circumstellar evolution, i.e. the formation of a detached shell from the interaction of different wind phases. The reason why we split up the modelling in this way, is the very different time and length scales of the relevant physical processes involved. An "all-in-one" model would demand unreasonable computing power in order to resolve the smallest lenght scales and the shortest time scales. In this section we describe the models and how the computations are carried out.

\subsection{Dynamic atmospheres}

The 1D (spherically symmetric) RHD code predicts the mass-loss rates of TP-AGB stars by detailed modelling of the atmosphere and the circumstellar environment around these pulsating long-period variable stars. The model includes frequencydependent radiative transfer and dust formation, i.e. we are solving the coupled system of frequency-dependent radiation hydrodynamics and time-dependent dust formation (cf. Höfner et al. 2003) employing an implicit numerical method and an adaptive grid.

In our model the stellar atmosphere and circumstellar envelope are described in terms of conservation laws for the gas, the dust and the radiation field, i.e. we solve the following set of coupled, nonlinear partial differential equations (PDEs):

- The three equations describing conservation of mass, momentum and energy for the gas.

- The 0th and 1st moment equation of radiative transfer. 
Table 1. Schematic overview of the model. Radial and time scales given are approximate figures.

\begin{tabular}{lccccl}
\hline \hline Model & Input & Output & Radial scale & Time scale & Comment \\
\hline Stellar evolution & $M_{\mathrm{ZAMS}}, Z$ & $M_{\star}, L_{\star}, T_{\mathrm{eff}}, \mathrm{C} / \mathrm{O}$ & $R \leq R_{\star}$ & $\sim 10^{4} \mathrm{yr}$ & Pre-computed \\
Atmosphere / wind & $\begin{array}{c}M_{\star}, L_{\star}, T_{\mathrm{eff}}, \\
\mathrm{C} / \mathrm{O}, P, \Delta v_{\mathrm{p}}\end{array}$ & $\dot{M}, v_{\text {wind }}(r)$ & $R_{\star}<R \leq 25 R_{\star}$ & $\sim 10^{2} \mathrm{yr}$ & $\begin{array}{l}\text { Full RHD + dust, } \\
\text { "snap shots" of evolution }\end{array}$ \\
CSE evolution & $\dot{M}, v_{\text {wind }}$ & $\rho_{\mathrm{env}}(r), v_{\mathrm{env}}(r)$ & $25 R_{\star}<R_{\mathrm{s}} \leq 10^{4} R_{\star}$ & $\sim 10^{4} \mathrm{yr}$ & $\begin{array}{l}\text { Gas dynamics, } \\
\text { dust dynamics not considered }\end{array}$ \\
\hline
\end{tabular}

- The four moment equations of dust formation (cf. Sect. 2.3).

- The Poisson equation (self gravity).

To this system of nonlinear partial differential equations we add a "grid equation" which determines the locations of the grid points according to accuracy considerations (Dorfi \& Drury 1987) and an equation keeping track of the condensable amount of carbon, leaving us with a total of 12 partial differential equations (PDEs) to solve. This system of PDEs is then solved implicitly using a Newton-Raphson scheme. All equations are discretised in a volume-integrated conservation form on a staggered mesh. The spatial discretisation of the advection term is a monotonic second-order advection scheme (van Leer 1977). The same order of numerical precision is used for all PDEs. Details of the numerical method are discussed by Dorfi \& Feuchtinger (1995) and in several previous papers about dust-driven wind models (cf. Höfner et al. 1995, and references therein).

The dynamical calculations are carried out as follows. All wind models are started from hydrostatic dust-free initial models where the outer boundary is located close to the photosphere (about $1.3-1.6 R_{\star}$ ). In these initial models, the fundamental stellar parameters $\left(M, L_{\star}, T_{\text {eff }}, \mathrm{C} / \mathrm{O}\right)$ can be chosen freely as inital conditions for the models. In the present case we pick the sets of stellar parameters from an evolutionary track as described in Sect. 2.1. When the dust equations are switched on, dust formation starts and creates an outward motion of the dust and the gas. In the first computational phase the expansion of the atmospheric layers is followed by the grid to about $20-30 R_{\star}$ (usually $\sim 10^{15} \mathrm{~cm}$ ). At this radius the location of the outer boundary $R_{\text {out }}$ is fixed allowing outflow. The outflow model then evolves for typically more than 200 years, but the precise time is dependent on the pulsation period. The amount of mass between the inner boundary $R_{\text {in }}$ and the outer boundary $R_{\text {out }}$ is roughly $10^{-2} M_{\odot}$, which is sufficient to avoid any significant mass depletion within the time series considered here.

The model is self-consistent as far as the physics of the stellar atmosphere is concerned, but it does not include a physical model for the pulsation mechanism. The pulsation is modelled phenomenologically using a "piston boundary condition" (Bowen 1988) located at $R_{\text {in }} \approx 0.9 R_{\star}$. To restrict the number of free parameters, we employ an empirical period-luminosity relation (Feast et al 1989) and keep the period locked to the luminosity for all models. The piston amplitude, however, is essentially a free parameter that we cannot do away with as easily. For this reason we must consider the effects of picking different amplitudes, which is a complication that we will get back to in Sect. 4.1. The motion of the piston is accompanied by luminosity variations, since the flux through the lower boundary is kept fixed (as described in previous papers, e.g., Höfner \& Dorfi 1997). Note, however, that assuming no artificial scaling of the short-term luminosity variations due to the pulsations, these are more than a factor of two weaker than the long-term luminosity variation due to the flash.

\subsection{Dust formation and mass loss}

Mass loss from TP-AGB carbon stars is mainly due to momentum transfer from radiation to atmospheric dust grains. This is often referred to as a dust driven wind. As such, a wind model for TP-AGB stars is highly dependent on how dust formation is treated. Hence, we will give a somewhat more detailed description of the dust model.

The models presented here include a time-dependent description of dust grain growth and evaporation using the moment method by Gail \& Sedlmayr (1988) and Gauger et al. (1990). The dust component is described in terms of moments $K_{j}$ of the grain size distribution function weighted with a power $j$ of the grain radius. The zeroth moment, $K_{0}$, is the total number density of grains (simply the integral of the size distribution function over all grain sizes), while $K_{3}$ is proportional to the average volume of the grains. The equations, which determine the evolution of the dust component, are

$\frac{\partial K_{0}}{\partial t}+\nabla \cdot\left(K_{0} v\right)=\mathfrak{J}$

$\frac{\partial K_{j}}{\partial t}+\nabla \cdot\left(K_{j} v\right)=\frac{j}{3} \frac{1}{\tau} K_{j-1}+N_{\ell}^{j / 3} \mathfrak{J}, \quad 1 \leq j \leq 3$,

where $v$ is the flow velocity of the dust component, $\mathfrak{J}$ is the net grain formation rate per volume, $1 / \tau$ is the net growth-rate of the dust grains, and $N_{\ell}$ is the lower size limit of grains contributing to $K_{j}$.

In order to calculate how much of the radiative energy and momentum is transferred to dust grains, we need to know the frequency-dependent opacity of these grains. This can be expressed in terms of the extinction efficiency $Q_{\text {ext }}$, which is the ratio of the extinction cross section to the geometrical cross section of the grains. Since the grains are usually small compared to the wavelengths, $Q_{\text {ext }}$ becomes a simple function of the grain radius. The wavelength and grain size dependence of the opacity can thus be separated into two independent factors, which greatly simplifies the calculations. The models in this paper are calculated using the data of Rouleau \& Martin (1991) for $Q_{\text {ext }}$ (see Andersen et al. 2003; and Höfner et al. 2003, for further discussion). The intrinsic dust density used in the model is set to $\rho_{\mathrm{d}}=1.85 \mathrm{~g} \mathrm{~cm}^{-1}$, which matches the material in Rouleau \& Martin (1991).

We assume spherical dust grains consisting of amorphous carbon only, since the star we are modelling is a carbon star (see Andersen et al. 2003 for details about these assumptions). The nucleation, growth and evaporation of grains is assumed to proceed by reactions involving $\mathrm{C}, \mathrm{C}_{2}, \mathrm{C}_{2} \mathrm{H}$ and $\mathrm{C}_{2} \mathrm{H}_{2}$. In this model of grain growth, a so called sticking coefficient (sometimes referred to as the reaction efficiency factor) is used, that enters into 
the net growth rate of the dust grains. This parameter, $\alpha_{\mathrm{S}}$, is not definitely known unless we know the exact sequence of chemical reactions responsible for the dust formation. However, Gail \& Sedlmayr (1988) argued that the sticking coefficient must be on the order of unity, mainly because it is expected that neutral radical reactions play a major role in the formation of carbon grains. It should be pointed out as well, that with $\alpha_{\mathrm{S}}=1$, our models nicely reproduce the expected mass loss properties of TP-AGB stars (Schöier et al. 2001; Schöier et al. 2005). See Sect. 4.1 for some further details.

Dust grains in a stellar atmosphere influence both its energy and momentum balance. For simplicity we assume complete momentum and position coupling of gas and dust, i.e. the momentum gained by the dust from the radiation field is directly transferred to the gas and there is no drift between dust and gas. However, this strong coupling between the dust and the gas phase is not obvious. In a previous attempt to relax this phase coupling approximation, Sandin \& Höfner $(2003,2004)$ found that the effects of decoupling of the phases might be quite significant. The most striking feature is that the dust formation may increase significantly, but this does not seem to necessarily increase the predicted mass loss rates for a given set of stellar parameters. However, these models are not directly comparable with observations, since one essential component - frequency dependent radiative transfer - is not included. Comparison with observations (Gautschy-Loidl et al. 2004; Nowotny et al. 2005a,b) seems to indicate that in general the inclusion of frequency-dependent radiative transfer is crucial for a realistic description of the pulsating amosphere, and therefore for the conditions in the wind formation region. A coupled solution of the detailed frequencydependent equations of RHD, including dust formation and drift would lead to a prohibitive computational effort and is not a realistic option in the present context. Thus, we chose to give frequency-dependent radiative transfer priority over relaxing the phase coupling approximation, even if this approximation cannot hold for very low gas densities, i.e. at large distances from the star or for models with low mass loss rates. Furthermore, since transfer of internal energy between gas and dust is negligible compared to the interaction of each component with the radiative field (Gauger et al. 1990), we assume radiative equilibrium for the dust. This allows us to estimate the grain temperatures from the radiation temperature as we know the dust opacities.

From the models described in this section and in 2.2 we obtain the density and the wind velocity, both as functions of radius and time. The mass loss rate is then given by

$\dot{M}=4 \pi R_{0}^{2} \rho_{0}(t) v_{0}(t)$,

where $\rho_{0}$ and $v_{0}$ are the density and wind velocity at $R_{0}$, i.e. the radius where the outer boundary is fixed, which is usually around $10^{15} \mathrm{~cm}$ and enough to ensure that the wind has reached the terminal velocity. Since practically all momentum transfer from radiation to matter is due to the interaction with dust, we expect the wind velocity to be correlated with the degree of dust condensation. Thus, Eq. (3) leads to the expecation that under most circumstances a larger proportion of dust will correspond to a higher mass loss rate.

\subsection{Stellar evolution}

To model how the atmosphere evolves on longer time scales (significantly longer than the pulsation period) due to changes in the internal constitution and nuclear processes of the star, we have pre-computed a stellar evolutionary track (see Fig. 1). The stellar parameters are then sampled at critical or characteristic points (tick-marked in the lower part of each panel in Fig. 1) around a thermal pulse in the evolutionary track and used as input to the atmosphere model. In total we pick 21 points along the track that we know from experience will capture the important features. By computing a set of models in this way for different points in the time evolution, we can obtain the change of atmospheric properties and wind characteristics (i.e. mass loss rate and wind velocity) during the flash event.

The calculations have been done with the one-dimensional stellar evolution code EVOL (Herwig 2000, 2004, and references therein). We use the EVOL implementation of exponential, time and depth dependent overshooting, here with an efficiency $f=0.016$ at the bottom of the convective envelope, but no overshooting at the bottom of the He-shell flash convection zone. EVOL features updated micro-physics input, including a nuclear reaction network. The Schwarzschild criterion for convection is used and the mixing length parameter is set to $\alpha_{\mathrm{MLT}}=1.7$. Mass loss during AGB evolution is included as prescribed by Blöcker (1995). Specifically we use Eq. (11) from that work in which $\Gamma(M)$ is determined according to his Eqs. (13) and (14). For the mass in Eq. (13) the intial ZAMS mass is assumed as an approximation of the actual mass at which the oscillation period is $P_{0}=100 \mathrm{~d} . \dot{M}_{R}$ in Eq. (11) is the Reimers mass loss rate (Reimers 1975) as given by Eq. (1) in Blöcker (1995). The scaling factor in that equation is set to $\eta_{\mathrm{R}}=0.1$, and gives an overall evolution which is consistent with many obserational contraints, e.g., the number of thermal pulses expected after transformation into a carbon star.

For this detailed study we have picked the last but one (the 17th out of 18) thermal pulse of sequence ET13 (Herwig et al. 2006). This sequence has the metalicity $Z=0.01$ and an initial mass of $2 M_{\odot}$. At the time of the selected thermal pulse the total mass is $1.22 M_{\odot}$ and the core mass is $0.59 M_{\odot}$. The third dredge-up is well developed after the selected thermal pulse, with an efficiency of $\lambda=0.154$, after $\lambda=0.372$ at the previous pulse. Dredge-up efficiency decreases at the end of the TP-AGB evolution when the envelope mass decreases.

To minimise the number of atmosphere models needed to obtain the time evolution, we assume a constant stellar mass $\left(M_{\star}=1.2 M_{\odot}\right)$ during the thermal pulse. This is a reasonable simplification in this context even if the stellar mass will decrease notably on longer time scales due to the steady mass loss before the first flash and in between subsequent flashes (see Fig. 1, which captures the flash event we have modelled). This assumption will enable us to use some models at more than one sampling point, since in contrast to, e.g., $T_{\text {eff }}$, the stellar mass $M_{\star}$ is not a very critical parameter in the wind and CSE models. For the luminosity and temperature this "recycling" of models leads to deviations from the evolutionary track no larger than $1 \%$. Exact matching of stellar parameters will not increase the accuracy of the output, since small changes $(<1 \%)$ in the input parameters have virtually no effect on the resulting wind and mass loss rate. We also assume a constant $\mathrm{C} / \mathrm{O}$ ratio (where $\log \left(\varepsilon_{\mathrm{O}}\right)=-3.35$ ) before and after the flash event, where $\mathrm{C} / \mathrm{O}=1.85$ before the flash, and $\mathrm{C} / \mathrm{O}=2.01$ during and after the flash. In practice, this is really not an approximation, since on an evolutionary time scale the carbon abundance in the atmosphere makes a sudden jump from one basically constant level to another when a He-shell flash takes place. The numbers given above correspond to the evolutionary track we have computed. 

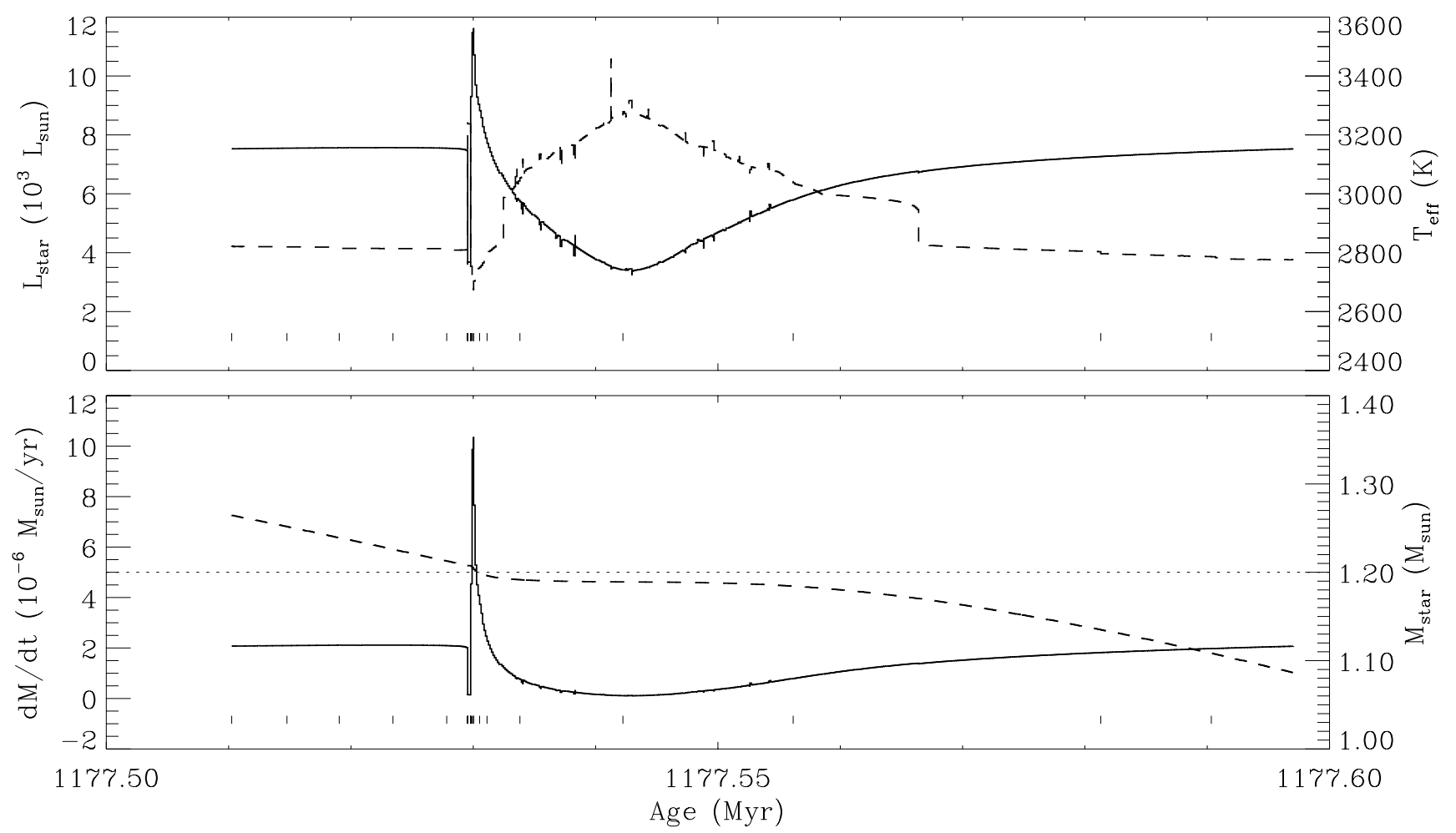

Fig. 1. Time evolution of luminosity $L_{\star}$ (upper panel, full line), effective temperature $T_{\text {eff }}$ (upper panel, dashed line), mass loss rate $\mathrm{d} M / \mathrm{d} t$ assumed in the evolution model (lower panel, full line) and stellar mass $M_{\star}$ (lower panel dashed line). The sampling points for the stellar parameters used as input to the dynamical atmosphere model are tick-marked in the lower part of each panel and the dotted line in the lower panel indicates the stellar mass that we adopt for the atmosphere models. The jump in $T_{\text {eff }}$ seen near $t=1177.58 \mathrm{Myr}$ is a numerical artifact.

\subsection{CSE evolution}

Since the mass loss rate and wind velocity are changing with time, so are the boundary conditions for the modelling of the CSE around the star (the region where the detached shell is formed). The inner boundary conditions for such a model, i.e. wind velocity $v_{0}$ and density $\rho_{0}$ at $R_{0}$ (the radius where the mass loss is obtained) as functions of time, are defined by interpolating the time sequence of atmosphere models computed as described above. Here, we are interested in whether these time variations will lead to the formation of a detached shell. The next step is therefore to compute the dynamical CSE evolution subject to such variable boundary conditions.

Some combinations of stellar parameters and piston amplitudes $\Delta v_{\mathrm{p}}$ will result in models without any mass loss (see Table 2). For such cases - if they occur in the post-flash phase - we assume a minimal mass loss rate of $10^{-8} M_{\odot} \mathrm{yr}^{-1}$, which is motivated by observations (Olofsson et al. 1998; Schöier et al. 2001, 2005), and estimate the mimimum wind velocity to be $\sim 1 \mathrm{~km} \mathrm{~s}^{-1}$. The circumstellar density at $R_{0}$ can thus be estimated using Eq. (3). Another possibility would be to simply take the average density at $R_{0}$ from the dynamical model with the the outer boundary radius $R_{0}$ set closer $\left(\sim 1.5 R_{\star}\right)$ to the photosphere and assume a minimal mass loss rate, say $10^{-8} M_{\odot} \mathrm{yr}^{-1}$. Then the wind velocity can be estimated through Eq. (3). We prefer the first method, where we set $v_{0}=1 \mathrm{~km} \mathrm{~s}^{-1}$, although both methods give more or less the same numbers. In the transition region between intense dust-driven mass loss and no mass loss, we set the rate to $10^{-7} M_{\odot} \mathrm{yr}^{-1}$ and assume the wind velocity to be $3 \mathrm{~km} \mathrm{~s}^{-1}$ and compute the density as described above. The exact numbers are not important in this case, since these models represent a phase with essentially no stellar wind that occurs after the He-shell flash.
We employ a simplified model of the CSE evolution where the equations of continuity and motion are given by

$$
\begin{aligned}
& \frac{\partial \rho}{\partial t}+\nabla \cdot(\rho v)=0 \\
& \frac{\partial \rho v}{\partial t}+\nabla \cdot(\rho v v)=-\nabla P_{\mathrm{gas}},
\end{aligned}
$$

where $\rho$ is the mass density, $v$ is the velocity and $P_{\text {gas }}$ is the thermal gas pressure. These equations are solved using a simplified setup of the dynamic atmosphere code (radiative transfer and dust equations switched off), i.e., the numerical method is the same. We assume that the gravitational pull from the star does not affect the expansion of the CSE on the typical distance from the star considered here $\left(R \geq 10^{15} \mathrm{~cm}\right)$, since at this radius it is orders of magnitude weaker than in the atmospheric layers. The self-gravity of the CSE can also be considered negligible as the typical mass density is too low to produce any sigificant self-gravity effects. We also assume that the radiation field is dominated by the central star, i.e. a point-source field falling off as $\sim r^{-2}$. Hence, at large distances from the central star, neither gravity, nor radiation, will be a force that really matters. This is of course a somewhat simplistic approach, but it is certainly a fair approximation of the physical situation in the present case. The pressure gradient, however, is kept in the equation of motion, since it may play an important role in shock waves occuring at the interfaces of different wind phases.

Regarding the temperature structure, we consider two limit cases.

I: No radiative heating or cooling, the temperature structure is set by the expansion of the gas, leading to a fast decrease of 
Table 2. Input parameters $\left(L_{\star}, T_{\text {eff }}, \mathrm{C} / \mathrm{O}, P, \Delta v_{\mathrm{p}}\right)$ and the resulting mean mass loss rate, mean velocity at the outer boundary and mean degree of dust condensation at the outer boundary. $R_{\star}$ is the stellar radius of the hydrostatic initial model (obtained from $L_{\star}$ and $\left.T_{\text {eff }}\right)$ and $\log (g)$ is the corresponding surface gravity. The dust-to-gas mass ratio $\rho_{\text {dust }} / \rho_{\text {gas }}$ is calculated from $f_{\mathrm{c}}$ as described in Höfner $\&$ Dorfi (1997). All models have a mass of $M_{\star}=1.2 M_{\odot}$ and $\log \left(\varepsilon_{\mathrm{O}}\right)=-3.35$. The mean values of the wind properties in this table are all taken over at least 100 pulsation periods, which is sufficient to ensure a small error due intermittent variations of these quantities.

\begin{tabular}{|c|c|c|c|c|c|c|c|c|c|c|c|}
\hline Model & $\begin{array}{c}L_{\star} \\
{\left[L_{\odot}\right]}\end{array}$ & $\begin{array}{l}T_{\text {eff }} \\
{[\mathrm{K}]}\end{array}$ & $\begin{array}{c}R_{\star} \\
{\left[R_{\odot}\right]}\end{array}$ & $\overline{\log (g)}$ & $\overline{\mathrm{C} / \mathrm{O}}$ & $\begin{array}{c}P \\
\text { [days] }\end{array}$ & $\begin{array}{c}\Delta v_{\mathrm{p}} \\
{\left[\mathrm{km} \mathrm{s}^{-1}\right]}\end{array}$ & $\begin{array}{c}\langle\dot{M}\rangle \\
{\left[M_{\odot} \mathrm{yr}^{-1}\right]}\end{array}$ & $\begin{array}{c}\left\langle v_{\text {ext }}\right\rangle \\
{\left[\mathrm{km} \mathrm{s}^{-1}\right]}\end{array}$ & $\overline{\left\langle\mathrm{f}_{\mathrm{c}}\right\rangle}$ & $\begin{array}{c}\frac{\rho_{\text {dust }}}{\rho_{\text {gas }}} \\
{\left[10^{-4}\right]}\end{array}$ \\
\hline $158 \mathrm{t} 305 \mathrm{c} 201 \mathrm{u} 2$ & 5800 & 3050 & 343 & -0.55 & 2.01 & 333 & 2.0 & - & - & - & - \\
\hline $158 \mathrm{t} 305 \mathrm{c} 201 \mathrm{u} 4$ & 5800 & 3050 & 343 & -0.55 & 2.01 & 333 & 4.0 & - & - & - & - \\
\hline $158 \mathrm{t} 305 \mathrm{c} 201 \mathrm{u} 6$ & 5800 & 3050 & 343 & -0.55 & 2.01 & 333 & 6.0 & - & - & - & - \\
\hline $180 \mathrm{t} 28 \mathrm{c} 185 \mathrm{u} 2$ & 8000 & 2800 & 485 & -0.85 & 1.85 & 436 & 2.0 & - & - & - & - \\
\hline $180 t 28 \mathrm{c} 185 \mathrm{u} 4$ & 8000 & 2800 & 485 & -0.85 & 1.85 & 436 & 4.0 & $6.0 \times 10^{-7}$ & 10 & 0.16 & 3.9 \\
\hline $180 \mathrm{t} 28 \mathrm{c} 185 \mathrm{u} 6$ & 8000 & 2800 & 485 & -0.85 & 1.85 & 436 & 6.0 & $3.8 \times 10^{-6}$ & 21 & 0.38 & 9.1 \\
\hline $180 t 28 c 201 u 2$ & 8000 & 2800 & 485 & -0.85 & 2.01 & 436 & 2.0 & - & - & - & - \\
\hline $180 t 28 c 201 u 4$ & 8000 & 2800 & 485 & -0.85 & 2.01 & 436 & 4.0 & $1.3 \times 10^{-6}$ & 15 & 0.18 & 10 \\
\hline $180 t 28 \mathrm{c} 201 \mathrm{u} 6$ & 8000 & 2800 & 485 & -0.85 & 2.01 & 436 & 6.0 & $2.8 \times 10^{-6}$ & 24 & 0.42 & 24 \\
\hline $190 \mathrm{t} 275 \mathrm{c} 201 \mathrm{u} 2$ & 9000 & 2750 & 530 & -0.93 & 2.01 & 481 & 2.0 & - & - & - & - \\
\hline $190 t 275 \mathrm{c} 201 \mathrm{u} 4$ & 9000 & 2750 & 530 & -0.93 & 2.01 & 481 & 4.0 & $2.8 \times 10^{-6}$ & 21 & 0.31 & 18 \\
\hline $190 t 275 c 201 u 6$ & 9000 & 2750 & 530 & -0.93 & 2.01 & 481 & 6.0 & $4.8 \times 10^{-6}$ & 24 & 0.42 & 24 \\
\hline $1105 \mathrm{t} 27 \mathrm{c} 201 \mathrm{u} 2$ & 10500 & 2700 & 654 & -1.11 & 2.01 & 547 & 2.0 & $1.1 \times 10^{-6}$ & 16 & 0.15 & 8.5 \\
\hline $1105 \mathrm{t} 27 \mathrm{c} 201 \mathrm{u} 4$ & 10500 & 2700 & 654 & -1.11 & 2.01 & 547 & 4.0 & $4.6 \times 10^{-6}$ & 17 & 0.24 & 14 \\
\hline $1105 \mathrm{t} 27 \mathrm{c} 201 \mathrm{u} 6$ & 10500 & 2700 & 654 & -1.11 & 2.01 & 547 & 6.0 & $6.7 \times 10^{-6}$ & 20 & 0.34 & 19 \\
\hline $112 \mathrm{t} 265 \mathrm{c} 201 \mathrm{u} 2$ & 12000 & 2650 & 682 & -1.15 & 2.01 & 611 & 2.0 & $3.0 \times 10^{-6}$ & 19 & 0.17 & 9.7 \\
\hline $112 \mathrm{t} 265 \mathrm{c} 201 \mathrm{u} 4$ & 12000 & 2650 & 682 & -1.15 & 2.01 & 611 & 4.0 & $7.1 \times 10^{-6}$ & 18 & 0.24 & 14 \\
\hline $112 \mathrm{t} 265 \mathrm{c} 201 \mathrm{u} 6$ & 12000 & 2650 & 682 & -1.15 & 2.01 & 611 & 6.0 & $9.9 \times 10^{-6}$ & 19 & 0.32 & 18 \\
\hline
\end{tabular}

temperature with distance from the star. Shocks are assumed to be adiabatic.

II: A fixed, given temperature structure only reflecting the radiation field of the star, leading to a more gentle dropping of temperature with distance. Shocks are isothermal relative to the surrounding medium.

More precisely, in Case I we are solving an energy equation of the form

$\frac{\partial \rho e}{\partial t}+\nabla \cdot(\rho e v)=-P_{\text {gas }} \nabla \cdot v$,

(where $e$ is the specific internal energy and all other variables are denoted as before) together with the equation of motion and the continuity equation (see above). This means that the energy equation used for the CSE differs from the full RHD version of the atmosphere and wind acceleration region by the lacking source and sink terms for radiative heating and cooling (cf., e.g., Höfner et al. 2003).

In Case II, representing the other extreme, we assume that the temperature structure is fully determined by radiative equilibrium with the stellar radiation field. In this case, we have

$\int_{0}^{\infty} \kappa_{v}\left(J_{v}-S_{v}\right) \mathrm{d} v=0$

where $\kappa_{v}$ is the monochromatic opacity, $J_{v}$ is the mean intensity and $S_{v}$ is the source function. Hence, the zeroth order moment equation of radiation,

$\nabla \cdot H+\rho\left(\kappa_{\mathrm{J}} J_{v}-\kappa_{\mathrm{S}} S\right)=0$,

where the frequency integrated opacities $\kappa_{J}$ and $\kappa_{S}$ are defined as in Höfner et al. (2003), implies that $H$ in such a case must be divergence free, e.g. like a point source field. We thus estimate the radiative field from

$J_{v} \approx W(r) B_{v}\left(T_{\star}\right)$

where

$W(r)=\frac{1}{2}\left[1-\sqrt{1-\left(\frac{R_{\star}}{r}\right)^{2}}\right] \approx \frac{1}{4}\left(\frac{R_{\star}}{r}\right)^{2}, \quad r \gg R_{\star}$,

is a geometrical dilution factor (cf. Lamers \& Cassinelli 1999). If we assume radiative equilibrium for dust, and $T_{\text {gas }} \approx T_{\text {dust }}$, we end up with the relation

$T_{\text {gas }}^{4} Q_{\mathrm{Pl}}\left(a, T_{\text {dust }}\right)=W(r) T_{\star}^{4} Q_{\mathrm{Pl}}\left(a, T_{\star}\right)$,

where $Q_{\mathrm{PI}}(a, T)$ is the Planck mean extinction efficiency as a function of the grain radius $a$ and the temperature. A simple estimate of the frequency dependence ${ }^{1}$ can be made from assuming a power-law relation $Q(a, v) \sim v^{n}$. In such a case,

$T_{\text {dust }}=W(r)^{1 /(4+n)} T_{\star}$,

which for $r \gg R_{\star}$ becomes

$T_{\text {dust }} \approx\left(\frac{R_{\star}}{r}\right)^{2 /(4+n)} T_{\star}$.

Considering amorphous carbon grains, a reasonable choice for the power index is $n \approx 1$, i.e. a linear dependence on frequency.

${ }^{1}$ If the monochromatic $Q$ is frequency-independent (gray case), the Planck mean $Q_{\mathrm{Pl}}$ is independent of $T$ and solving Eq. (10) for $T_{\text {dust }}$ gives $T_{\text {dust }} \sim T_{\star} r^{-1 / 2}$ for $r \gg R_{\star}$. Opacities are, however, frequency dependent and $Q_{\mathrm{Pl}}(a, T)$ is therefore not a constant. 

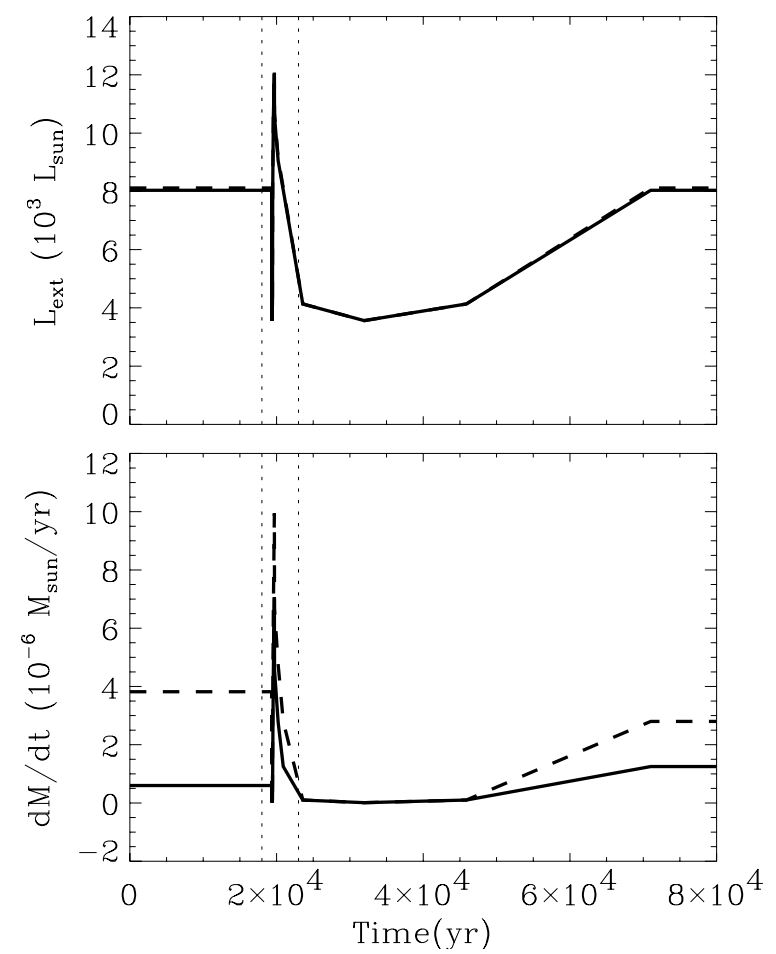
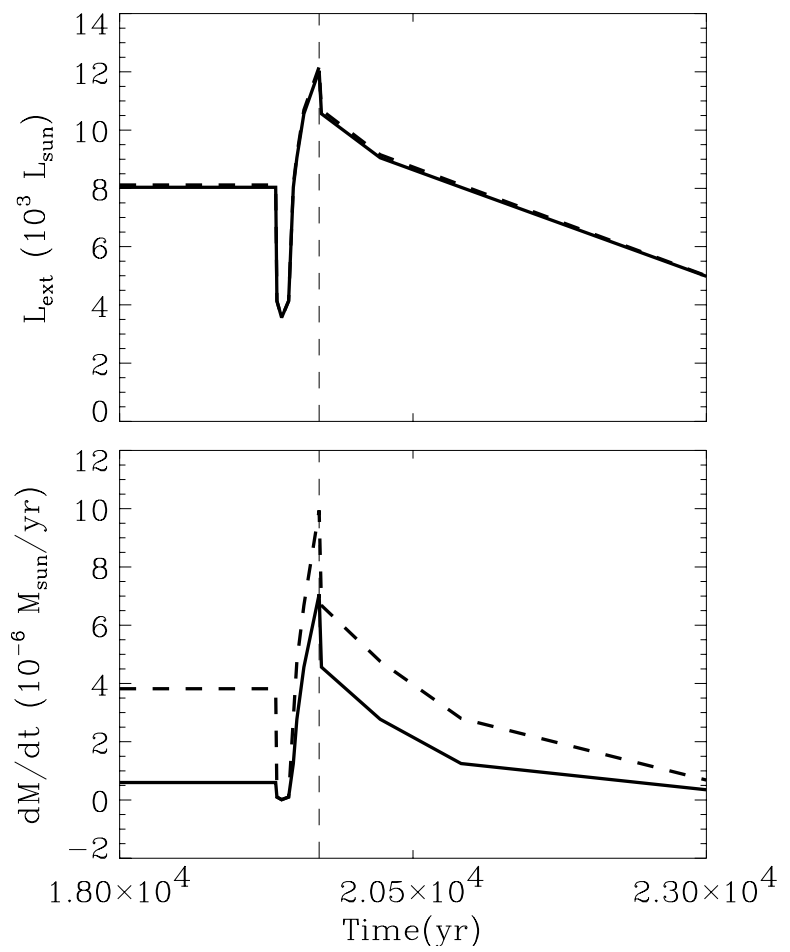

Fig. 2. Upper left panel: the external luminosity for $\Delta v_{\mathrm{p}}=4.0 \mathrm{~km} \mathrm{~s}^{-1}$ (full line) and $\Delta v_{\mathrm{p}}=6.0 \mathrm{~km} \mathrm{~s}^{-1}$ (dashed line) as a function of time. Lower left panel: the mass loss rate for $\Delta v_{\mathrm{p}}=4.0 \mathrm{~km} \mathrm{~s}^{-1}$ (full line) and $\Delta v_{\mathrm{p}}=6.0 \mathrm{~km} \mathrm{~s}^{-1}$ (dashed line) as a function of time. The right panels shows a close ups (the interval between the vertical dotted lines) demonstrating the effects of the thermal pulse. The vertical dashed line marks the luminosity peak of the He-flash.

For the strictly linear case we get $T_{\text {dust }} \sim T_{\star} r^{-2 / 5}$, which is consistent with the temperature structure found by SS2000 using their DEXCEL-code. Hence, we have replaced the energy equation in our CSE model by inserting this approximate temperature structure for the gas temperature

$T_{\mathrm{CSE}} \approx T_{0}\left(\frac{r}{R_{0}}\right)^{-2 / 5}$,

where $T_{0}=500 \mathrm{~K}$ and $R_{0}=7 \times 10^{14} \mathrm{~cm}$. This approximation is what we refer to as Case II, which is nearly the opposite to Case I, since the temperature structure is only reflecting the stellar radiation field and neither affected by shocks, nor by the expansion. It is reasonable to assume that $T_{\text {ad }} \leq T_{\text {gas }} \leq T_{\text {dust }}$, i.e., the true gas temperature structre will be somewhere in between the temperature structures implied by Case I and Case II.

\section{Results}

Stellar parameters and model results for the set of atmosphere models used to compute the mass loss history are presented in Table 2. A graphical display of the time evolution of the luminosity and the mass loss rate are shown in Fig. 2, where the expected correlation between these two quantities is evident. In Fig. 3 we compare the wind velocity and the mean degree of dust condensation - two quantities that show a correlation as well. In these plots, full lines represent the case where $\Delta v_{\mathrm{p}}=4.0 \mathrm{~km} \mathrm{~s}^{-1}$, while dashed lines correspond to $\Delta v_{\mathrm{p}}=6.0 \mathrm{~km} \mathrm{~s}^{-1}$. Increasing this piston amplitude leads to a much stronger pre-flash wind. The mass loss rate as well as the wind velocity changes significantly (as is evident from Figs. 2 and 3).

A close up of the evolution during the flash event (right panels in Figs. 2 and 3) reveals an anti-correlation between mass loss rate and wind velocity at the peak of the flash. This may seem curious, but can be explained by the fact that the relative abundance of dust is lower in the flash-peak model, i.e the model corresponding to the highest luminosity (see Table 2). A smaller abundance of dust (lower $\rho_{\text {dust }}$ ) will result in a less effective momentum transfer between radiation and matter. Hence, we could expect a lower wind velocity, but not necesserily a lower mass loss rate, since the latter is proportional to both the matter density of the atmosphere and the wind velocity. In the particular case of the flash-peak model the gas density is higher, which leads to a higher mass loss rate although the wind is slower. The correlation between the mean degree of dust condensation $f_{\mathrm{c}}$ and the mean wind velocity is seen in Fig. 3.

The fact that the mass loss rate, wind velocity and $f_{\mathrm{c}}$ are strongly dependent on the piston amplitude $\Delta v_{\mathrm{p}}$ means that the predicted wind and mass loss cannot be very precisely constrained. There is no way of knowing if this amplitude can be regarded as constant over time without a complete physical description of the pulsation mechanism. This is certainly an important remark in the present case, since it appears quite likely that the pulsation amplitude could change as the star goes through a He-shell flash. Furthermore, the difference in kinetic energy injection by the pulsations depending on the choice of $\Delta v_{p}$ may have significant effects on the amount of material being transferred out to the dust-formation region, and thus also for the degree of dust condensation and consequently for the momentum transfer and wind velocity. For a further discussion on constraints on $\Delta v_{\mathrm{p}}$, see Sect. 4.1 .

\subsection{Time evolution of the atmosphere}

Since the wind properties may show short-term, intermittent variations over time, the model output is time-averaged. The 

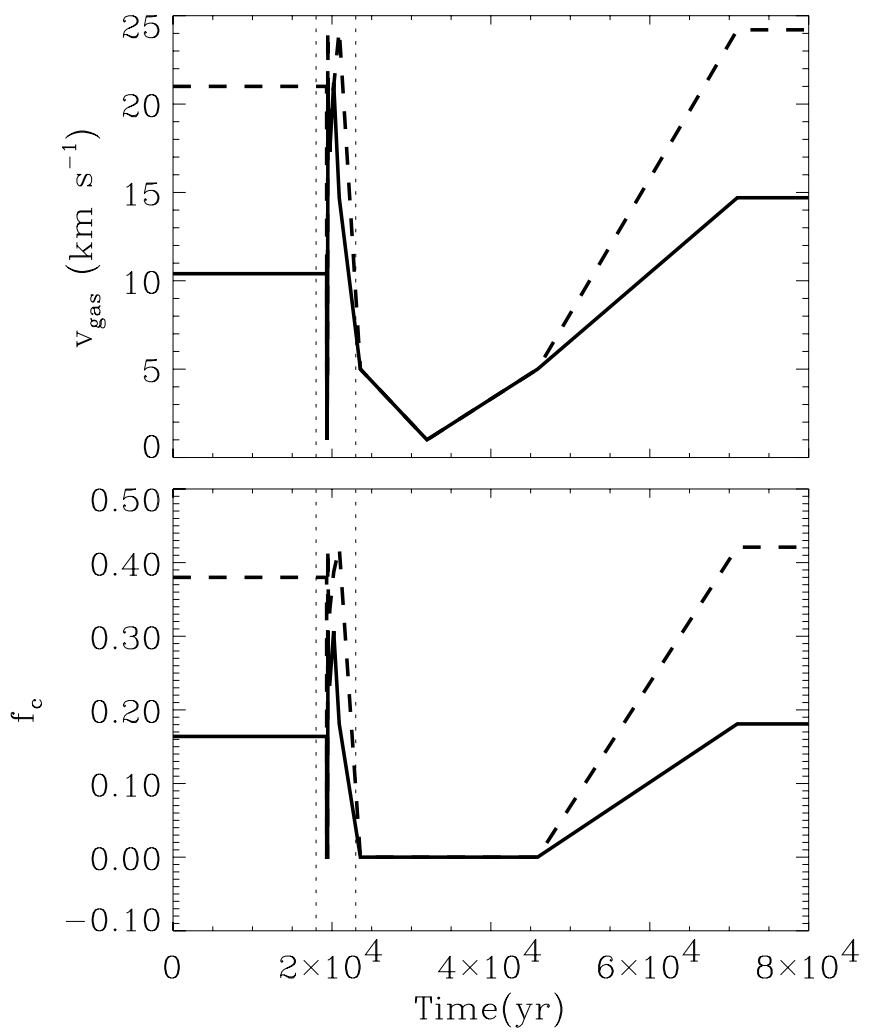
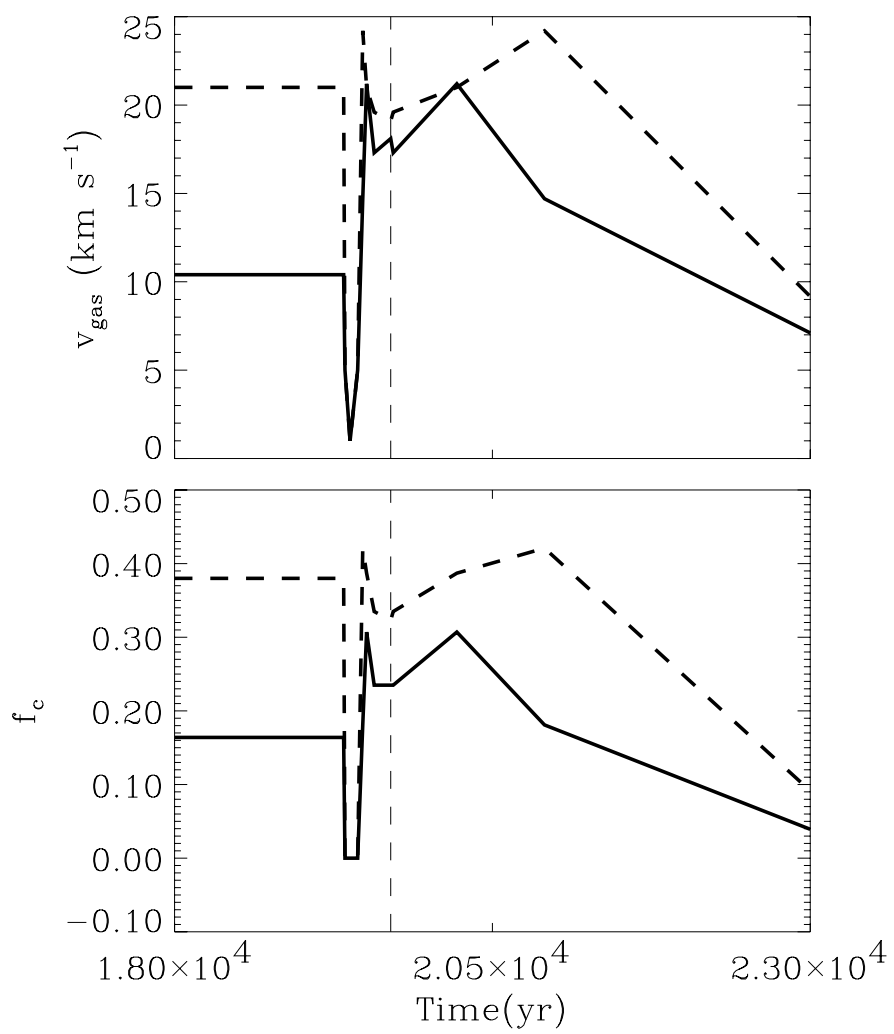

Fig. 3. Upper left panel: the wind velocity for $\Delta v_{\mathrm{p}}=4.0 \mathrm{~km} \mathrm{~s}^{-1}$ (full line) and $\Delta v_{\mathrm{p}}=6.0 \mathrm{~km} \mathrm{~s}^{-1}$ (dashed line) as a function of time. Lower left panel: the mean degree of dust condensation for $\Delta v_{\mathrm{p}}=4.0 \mathrm{~km} \mathrm{~s}^{-1}$ (full line) and $\Delta v_{\mathrm{p}}=6.0 \mathrm{~km} \mathrm{~s}^{-1}$ (dashed line) as a function of time. The right panels shows a close ups (the interval between the vertical dotted lines) demonstrating the effects of the thermal pulse. The vertical dashed line marks the luminosity peak of the He-flash.

models usually reach a statistical steady state after $\sim 50$ pulsation periods. An interval of $\sim 100$ periods is sufficient to obtain reliable mean values. A typical wind model is evolved over 150-200 periods, which is thus sufficient for the output to be statistically stable.

There is a complex interplay between density, dust formation, radiation pressure and gravity (escape velocity) that makes the variations in mass loss for different sets of stellar parameters highly nonlinear. This interplay is why simple parameterisations, where the mass loss rate is essentially just a function of the luminosity (e.g. Blöcker 1995; Wachter et al. 2002), cannot provide a satisfactory prescription for mass loss throughout the evolution of a star on the AGB. The well-known (but unfortunately often abused) Reimers formula (Reimers 1975) provides a mass loss prediction that cannot be made in agreement with the resulting mass loss evolution from our models. Regardless of the choice of the parameter $\eta_{\mathrm{R}}$ (in Fig. 4 the case $\eta_{\mathrm{R}}=1$ is shown) the mass loss evolution is not as strongly peaked at the time of the He-flash as the evolution implied by our models. However, Reimers formula was intended as a description of the mass loss from red giants, which does not include TP-AGB stars, and there are no justifications for extending the use of Reimers formula to this regime. Its linear dependence on the luminosity $L_{\star}$ means that it cannot at the same time reproduce the mass loss associated with the flash peak. A comparison of our results with existing mass loss formulae is presented Fig. 4, in which it becomes clear that none of the simple descriptions considered here agrees with our detailed model, nor are they consistent with each other. It is particularly interesting to notice that the description by Wachter et al. (2002) yields a significantly higher over-all mass loss rate compared with other prescriptions, as well as our wind model. The reason for this, as far as we can tell, is two-fold: Wachter et al. use grey radiative transfer (with lower gas opacities) which is known to give higher mass loss rates and they derive their formula in principle only from models with saturated dust-formation, i.e. models in the regime where the momentum transfer is most efficient.

In both Case I and Case II, this thin shell structure survives over the whole simulated CSE evolution. In Case II the thin shell structure remains quite intact as the shell expands - the only important change over time is a slight broadening of the density peak likely due to internal pressure. The evolution of Case I is somewhat different. Here a very pointed shell structure evolves shortly after flash event, but as the shell expands two shock fronts are formed, creating a density bump (or plateau) altough the initial density peak remains. The density peak is located at the contact discontinuity between the slow (pre-flash) and fast wind phases, where the gas temperature reaches a minimum.

\subsection{Formation of a detached shell}

The results of the hydrodynamic CSE evolution modelling confirm the "modified He-shell flash scenario" presented by SS2000 in the case of $\Delta v_{\mathrm{p}}=4.0 \mathrm{~km} \mathrm{~s}^{-1}$. Using the mass loss evolution we have obtained from our detailed modelling, we receive a density structure that is qualitatively consistent with the results of SS2000, but in general results in a much thinner shell. Case I also shows a time variation of the relative thickness not present in the results of SS2000 (see Fig. 9, Fig. 11 in this paper and Fig. 12 in SS2000). The reason for this time variation of the thickness is probably the different evolution of the velocity profile shown in our results, shortly after the He-shell flash. As the CSE evolves, 

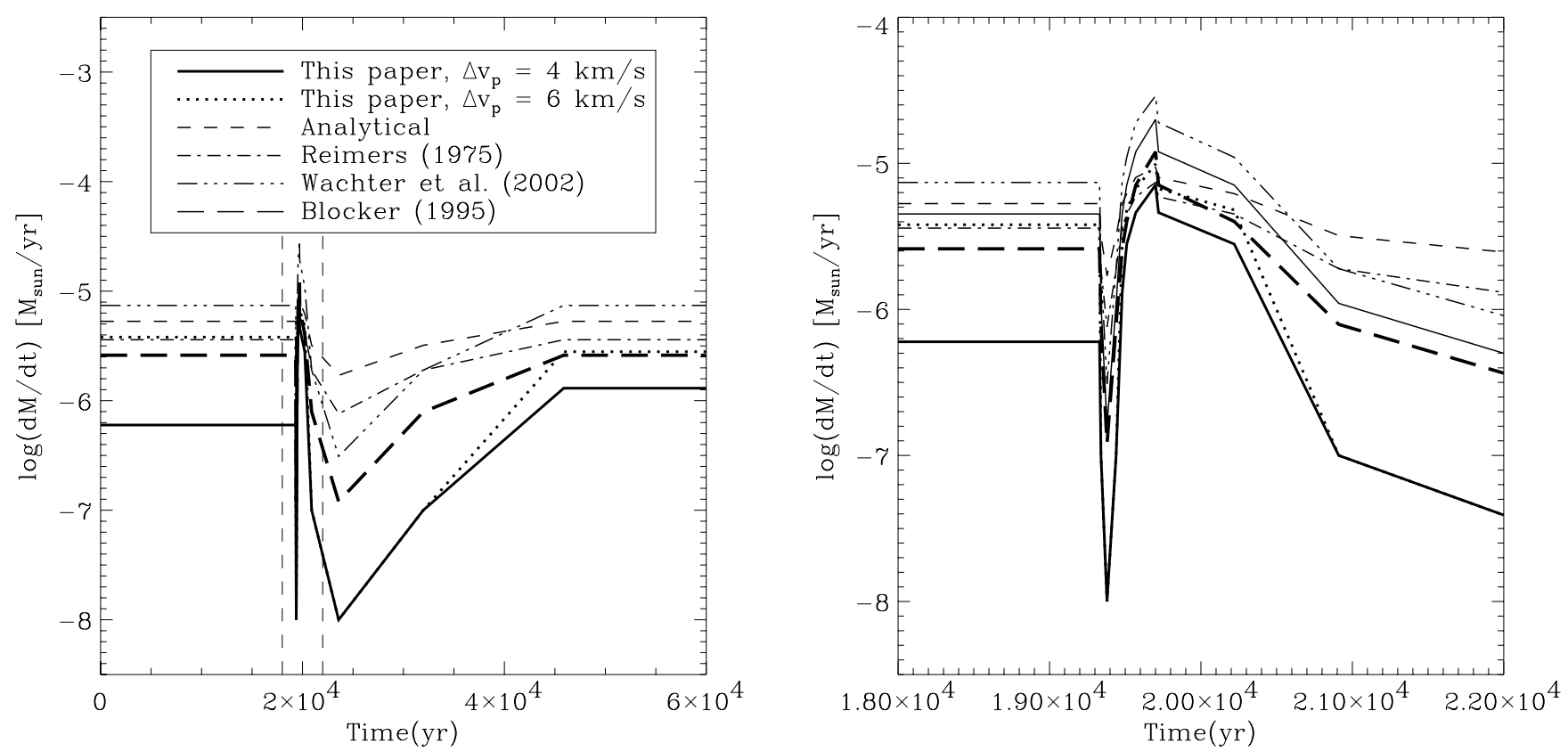

Fig. 4. Comparison with some existing mass loss formulae. The plot shows the mass loss evolution during the considered He-shell flash adopting mass loss rates from our atmosphere model (for two different values of $\Delta v_{\mathrm{p}}$ ), along with a simple analytic model (Lamers \& Cassinelli 1999), the empirical Reimers (1975) formula and the mass loss prescriptions derived from grids of numerical models by Wachter et al. (2002) and Blöcker (1995). Due to the large differences we have plotted the logarithm of the mass loss rate. The right panel shows a blow-up of the interval marked with vertical dashed lines in the left panel. Note as well, that for the evolution computed in this paper, the values at the mass loss minimum are ad hoc values.

the velocity profile becomes very similar to the result from the "modified He-shell flash scenario" presented in SS2000.

We have also calculated the FWHM of the quantity, $\mathrm{d} m / \mathrm{d} r=$ $4 \pi r^{2} \rho$, which we will refer to as the thickness of the shell. In Fig. 9 we show the time evolution of the thickness of the shell for Case I and II. As is evident from the plots, including a reasonable temperature structure has really a dramatic effect on the CSE evolution. Beyond $R=10^{17} \mathrm{~cm}$ (roughly 2000 years after the flash event) the relative thickness $\Delta R_{\mathrm{S}} / R_{\mathrm{S}}$ of the shell appears constant in Case II, while in Case I we see more of a $\Delta R_{\mathrm{S}} / R_{\mathrm{S}} \sim 1 / R_{\mathrm{S}}$ behaviour. The typical relative thickness in Case II is $\Delta R_{\mathrm{S}} / R_{\mathrm{S}} \sim 0.01$, which is less than what has been derived from observations (e.g. Olofsson et al. 2000). It should be pointed out, however, that these observations of detached shells we refer to are CO detections. González-Delgado et al. (2001) have detected shell structures around AGB stars in the optical, which have $\Delta R_{\mathrm{S}} / R_{\mathrm{S}} \sim 0.1$. This is an order of magnitude thicker than our model result and may indicate a problem with the assumptions made in Case II. In Case I the thickness is measured on the very sharp and pointed density peak (making the shell appear extremely thin), which means that the numbers we derive are only of interest for comparison with Case II.

The differences ocurring from the use of boundary conditions derived from atmosphere models with different piston amplitudes, are quite dramatic. With $\Delta v_{\mathrm{p}}=4.0 \mathrm{~km} \mathrm{~s}^{-1}$ the CSE model provides a pronounced shell structure, while the $\Delta v_{\mathrm{p}}=$ $6.0 \mathrm{~km} \mathrm{~s}^{-1}$ case does not lead to the formation of any shell structure at all (see Figs. 10 and 11). Since the $\Delta v_{\mathrm{p}}=2.0 \mathrm{~km} \mathrm{~s}^{-1}$ case was directly excluded (no wind, except at the flash peak) we are left with only one case that can produce a detached shell. In this case the density contrast at the shell radius is roughly two orders of magnitude relative to the surrounding medium, which should definitely be a detectable feature.
In order to determine if there are any effects on the shell formation from the short term variations of the wind during the flash (see Fig. 3), we have integrated the mass loss during the flash event and created a representative pulse function (same integrated mass loss) and replaced the time dependent inner boundary condition with this simplified function (see Fig. 8). The wind velocity is turned into a pulse function in the same fashion. Comparing the resulting CSE evolution with "case B" and "case C" in SS2000, it is clear that our CSE model produces a thinner and less massive shell, but still with an equally large density contrast. It is also evident that the small features (e.g. the dip at the flash peak) in the wind evolution found in our detailed modelling, have very little effect on the properties and evolution of the CSE and the detached shell (compare Figs. 10 and 11 with Figs. 12 and 13).

For the sake of completeness, we have tested how the mass loss history suggested by observations of TT Cygni (Olofsson et al. 1998; see also Figs. 1 and 4 in SS2000) affects the formation of a detached shell, using a boundary for the wind velocity which is equivalent to "case B" and "case C" in SS2000. As we show in Appendix A, the results we get for Case II are fully consistent with the results of SS2000 using their "NEBEL" code.

These tests elucidate the importance of modelling the wind evolution correctly. If the detailed inner boundary conditions derived from wind models are replaced by a combination of simple pulse functions (conserving differences in mass loss rate and wind velocity, before, during and after the flash), the CSE evolution is not much affected. If, on the other hand, the mass loss and velocity evolution differs as in "case B" and "case C" in SS2000, the CSE evolution will be different and results in a much thicker shell structure (e.g., compare Fig. 11 with Figs. A.2 and A.4). Thus, it is very important to use consistent combinations of mass 

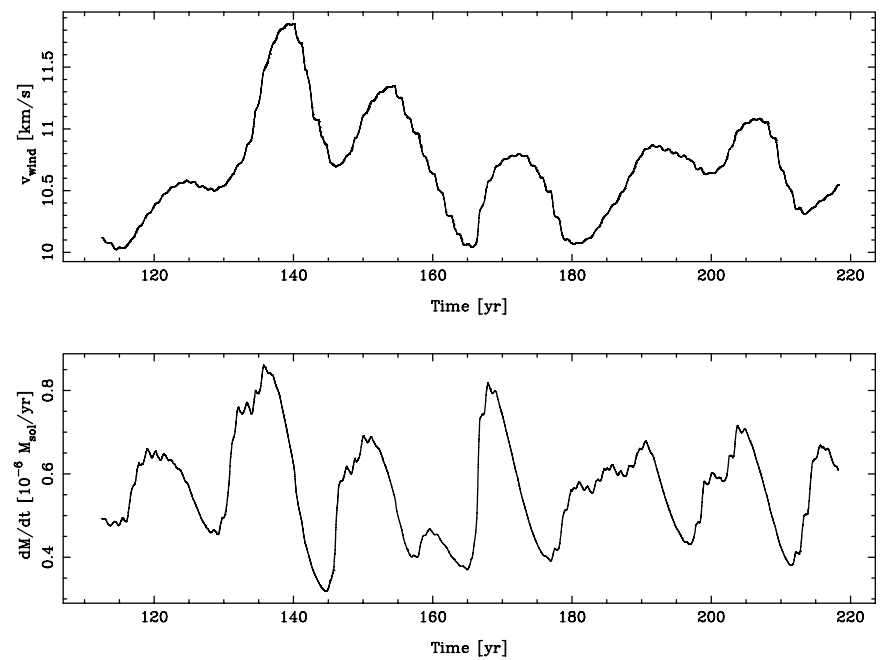

Fig. 5. Timeseries of the wind velocity and mass loss rate at $R_{0}$ showing the short-term variations before the He-flash (model $180 \mathrm{t} 28 \mathrm{~m} 12 \mathrm{c} 185$ ).
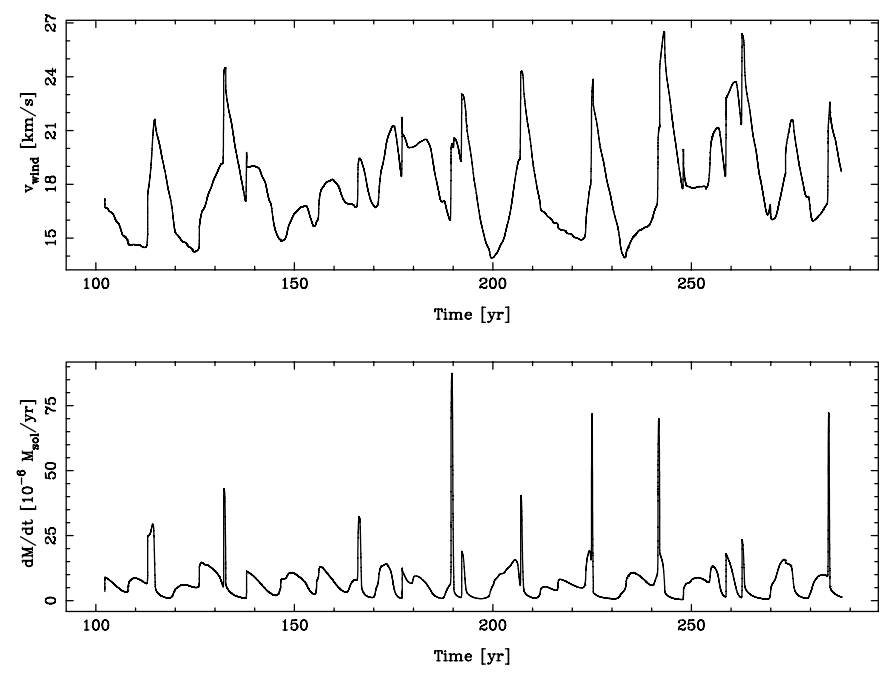

Fig. 6. Timeseries of the wind velocity and mass loss rate at $R_{0}$ showing short-term variations during the peak of the He-flash (model $112 \mathrm{t} 265 \mathrm{~m} 12 \mathrm{c} 201)$.

loss rates and wind velocities when modelling the evolution of the CSE.

\section{Discussion}

\subsection{Mass loss rates}

The pulsational instability in the stellar interior provides a mechanism for levitation of material into the outer atmosphere, i.e. the injection of kinetic energy from the pulsation related shock fronts as they propagate outwards may be sufficient to push material out to a radius where dust can begin to form. It is this phenomenon that enables the large mass loss rates associated with TP-AGB stars.

As described in Sect. 2.2 the pulsation is simulated by applying a piston boundary condition. Increasing the piston amplitude in the models from $\Delta v_{\mathrm{p}}=2.0 \mathrm{~km} \mathrm{~s}^{-1}$ to $\Delta v_{\mathrm{p}}=6.0 \mathrm{~km} \mathrm{~s}^{-1}$ can raise the density in the dust forming region by as much as one order of magnitude. Consequently, the amount of dust that forms may increase significantly as well and in turn lead to a more efficient momentum transfer and thus greater wind velocities. One
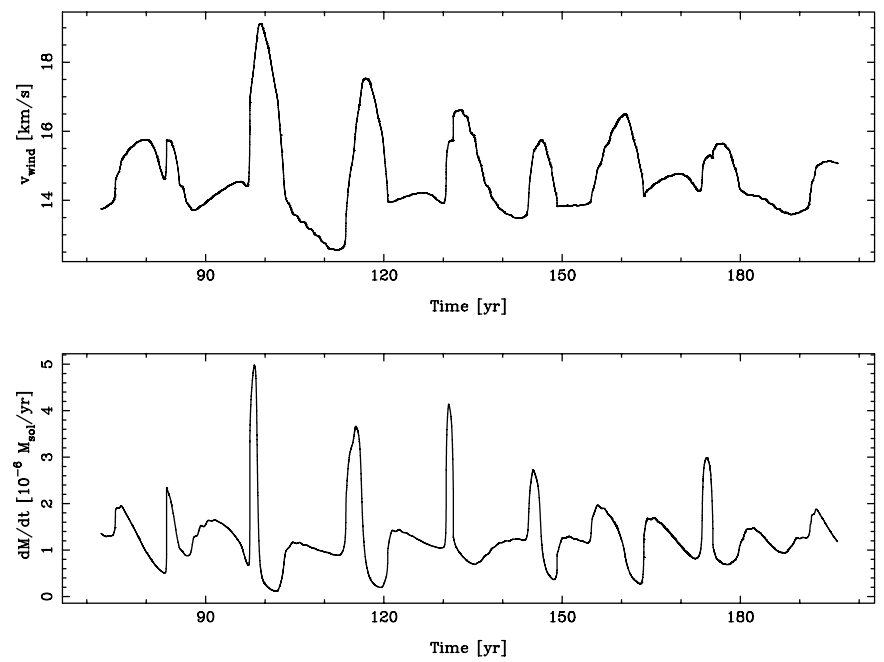

Fig. 7. Timeseries of the wind velocity and mass loss rate at $R_{0}$ showing short-term variations after the He-flash (model 180t28m12c201).

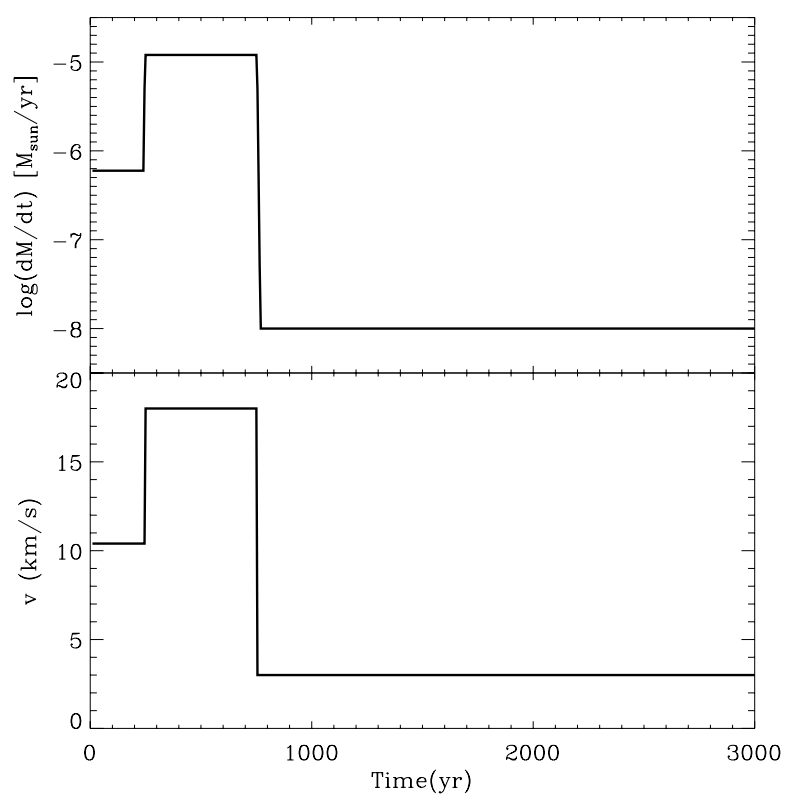

Fig. 8. Plots of the pulse functions used to replace the time dependent inner boundary condition.

can in princple fine tune $\Delta v_{\mathrm{p}}$ in order to get the wind properties that lead to shell formation for a specific set of stellar parameters. However, even if it is difficult to put strong constraints on the mass loss evolution without a reliable model for the pulsation mechanism, we can still limit the range of piston amplitudes that are reasonable in the present context. As we have seen, too small amplitudes do not lead to wind formation (except at the flash peak) and too large amplitudes will not provide the pronounced variations of wind properties needed to obtain the interacting wind phases that are crucial for shell formation. Hence, the possibility to choose the piston amplitude is restricted and we have found that the amplitude implied by previous comparsions with observations $\left(\Delta v_{\mathrm{p}}=4.0 \mathrm{~km} \mathrm{~s}^{-1}\right)$ is a good choice (e.g. Nowotny et al. 2005b). Furthermore, models with this amplitude give mass loss rates and wind velocities that matches observations of TP-AGB stars with known detached shells very well (e.g. Schöier et al. 2005). From recent observations of all objects with known detached molecular shells, Schöier et al. (2005) derived a post-flash mass loss rate (which at the same time gives 

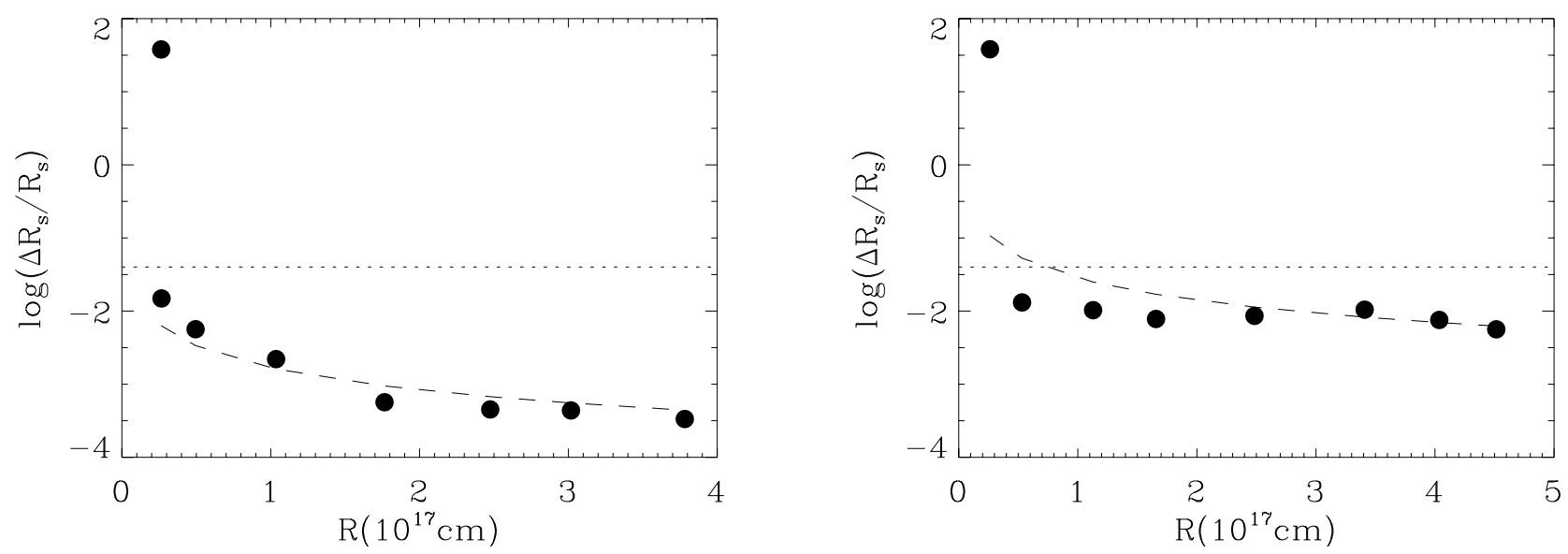

Fig. 9. Thickness of the detached shells formed in Cases I and II. The left panel shows Case I and the right panel shows Case II for $\Delta v_{\mathrm{p}}=4.0 \mathrm{~km} \mathrm{~s}{ }^{-1}$. The dashed lines indicate fits to $\Delta R_{\mathrm{S}} / R_{\mathrm{S}} \propto 1 / R_{\mathrm{S}}$. Each black dot represents a specific time in the evolution of the detached shell in accordance with all following plots of the velocity and density evolution. The dotted line marks the lower limit for the typical shell thickness derived by Olofsson et al. (2000) from CO observations.
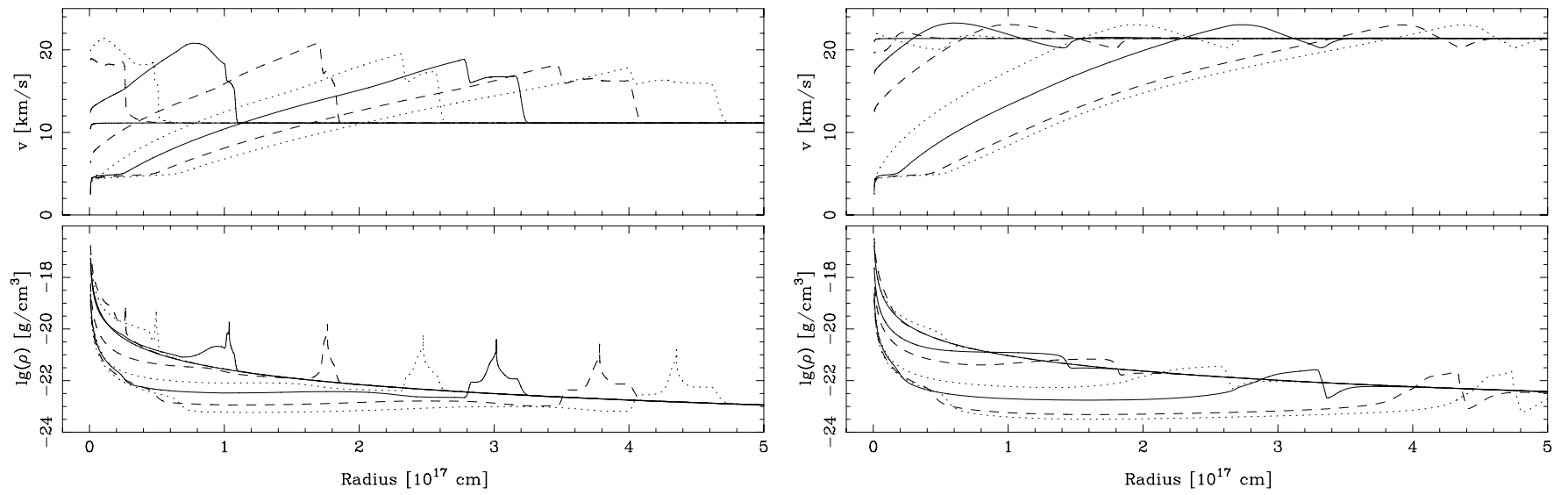

Fig. 10. Nine instants in the evolution of the CSE. Velocity and density structure of the CSE in Case I. Upper panels: the velocity field for $\Delta v_{\mathrm{p}}=4.0 \mathrm{~km} \mathrm{~s}^{-1}$ (left) and $\Delta v_{\mathrm{p}}=6.0 \mathrm{~km} \mathrm{~s}^{-1}$ (right). Lower panels: the logarithmic density for $\Delta v_{\mathrm{p}}=4.0 \mathrm{~km} \mathrm{~s}^{-1}($ left $)$ and $\Delta v_{\mathrm{p}}=6.0 \mathrm{~km} \mathrm{~s}{ }^{-1}($ right $)$.

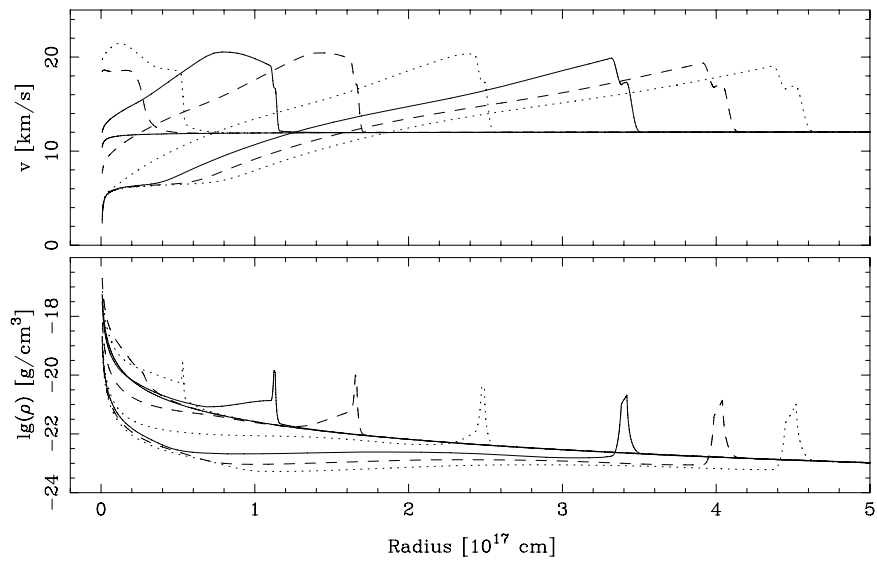

Fig. 11. Same as Fig. 10, but for Case II.

a hint about the pre-flash rate) of a few times $10^{-7} M_{\odot} \mathrm{yr}^{-1}$, and $\sim 10^{-5} M_{\odot} \mathrm{yr}^{-1}$ associated with a flash event, which is almost exactly what we get (see Fig. 2). The picture one gets is rather consistent - the pulsations are nicely modelled by a piston boundary condition with $\Delta v_{\mathrm{p}} \sim 4.0 \mathrm{~km} \mathrm{~s}^{-1}$ even if the physical mechanism is not modelled. We have thus not considered temporal variations of the pulsational amplitude during the flash event, even if they may occur.

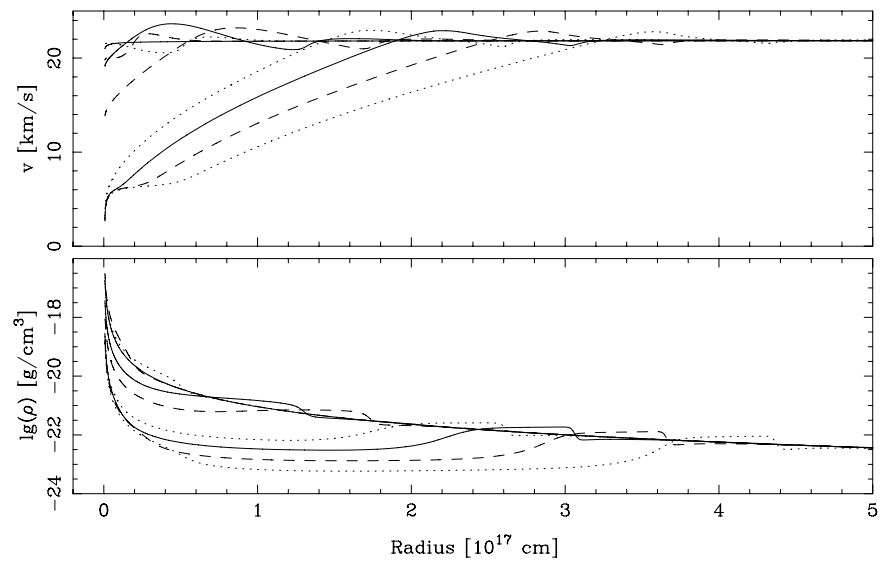

The three types of models that we have combined provide a rather consistent connection between a He-shell flash and detached shells with essentially one adjustable parameter, $\Delta v_{\mathrm{p}}$. However, there is one possible inconsistency that we have not mentioned so far. The evolutionary tracks for the present stellar model have been calculated using the mass loss prescription provided by Blöcker (1995). As shown in Fig. 4, the mass loss rates predicted by our model differ from most simple mass loss 


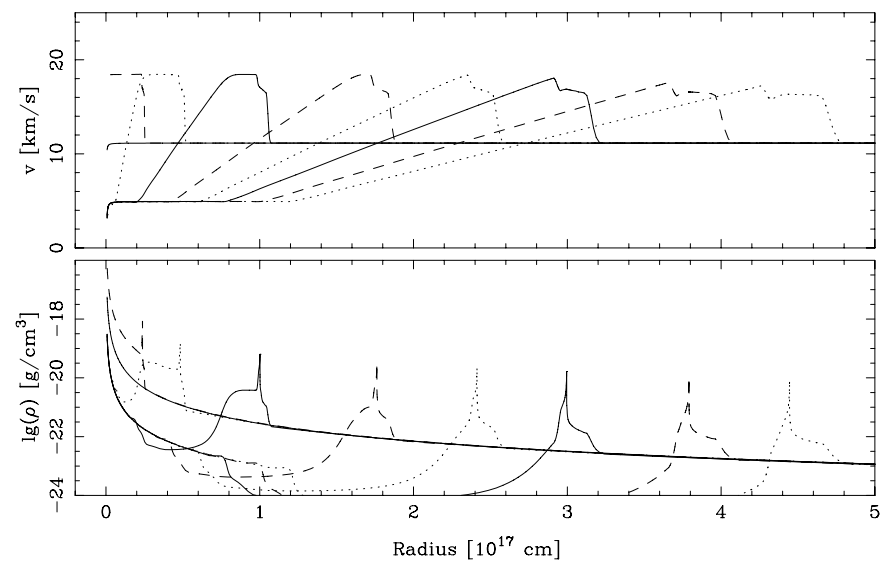

Fig. 12. Nine instants in the evolution of the CSE. Velocity and density structure of the CSE using the step function boundary condition (see Sect. 3.2) in Case I $\left(\Delta v_{\mathrm{p}}=4.0 \mathrm{~km} \mathrm{~s}^{-1}\right)$.

prescriptions (formulae). A significantly different mass loss evolution will alter the late stages of stellar evolution (as pointed out by Blöcker 1995) and thus provide a different set of stellar parameters as input to our model of the wind evolution. But this is most likely a minor problemns in terms of consistency, since the time interval we model is short on an evolutionary time scale and each one in the series of thermal pulses corresponds to slightly different sets of parameters, regardless of how the mass loss affects the stellar evolution. Moreover, the flash event itself, is not regulated by mass loss but by nuclear reaction rates. In our modelling we have picked one typical flash event that looked suitable in terms of getting a reasonable pre-flash mass loss rate, judging from previous experience of mass loss modelling.

\subsection{CSE evolution and the formation of a detached shell}

Let us now discuss how a detached shell may, or may not, be formed in the two limit cases of our CSE model (see Sect. 2.5) due to the wind evolution obtained from our wind models.

In Case I, where the global temperature structure is set by the expansion of the gas, the shock is basically adiabatic. The increase of the pressure leads to high temperatures (typically a few $10^{3} \mathrm{~K}$ ) in the shocked regions, which cannot be radiated away (since radiation is not included) and thus enforces a change in the gas motion that manifests itself by the build up of a shock wave in front of the ejected shell. This is why the shell evolves into a density bump with a density peak on top of it. There are several arguments against this kind of "adiabatic" evolution of the shell, where the most important one is that any shock front of this type must lose at least some energy through radiation. The high temperature we obtain inside the shocked regions as well as the low temperatures in the stationary pre-flash wind, indicate that Case I is quite unrealistic.

Case II, where we have assumed a fixed temperature structure, corresponds to having an isothermal shock front relative to its surroundings. This is in principle the opposite extreme of Case I, since the expanding shell is forced to have the same temperature as the surrounding medium, meaning that all excess energy is transferred away from the shock. Such an effectiveness in radiative cooling is quite unlikely, but Case II is probably still closer to reality than Case I - especially regarding the global temperature structure. To make a truly realistic model including all possible energy sinks and sources in this case, it is probably necessary to include a full RHD description (with

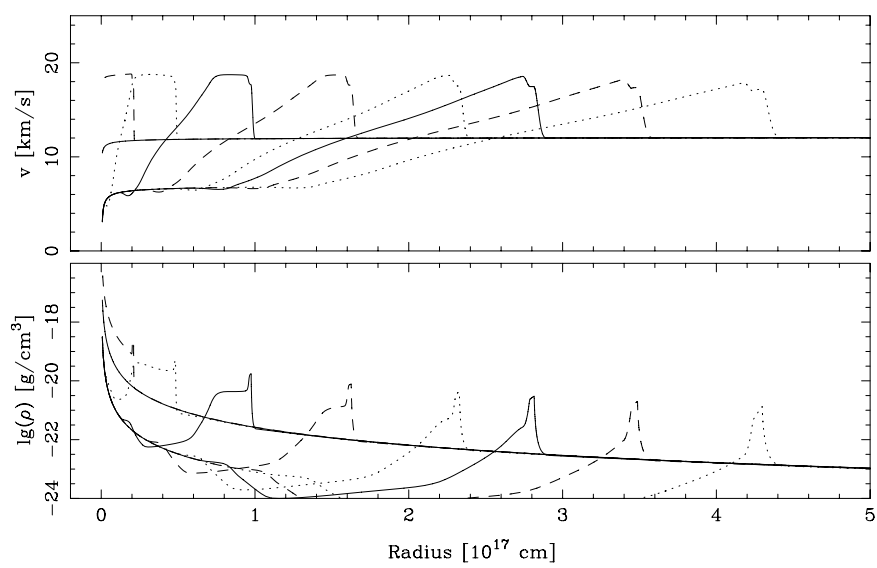

Fig. 13. Same as Fig. 12, but for Case II.

dust) of the problem and maybe even go from $1 \mathrm{D}$ to $3 \mathrm{D}$ in order to correctly treat turbulence and dynamical instabilities (see Myasnikov et al. 2000).

The reverse shock and the forward shock in front of the shell are effects due to the quite large velocity jumps that build up on each side of the shell as it propagates outward. This feature is most prominent in Case I, due to the overestimation of compressional heating and the resulting thermal pressure gradients.

As we pointed out in Sect. 3.2, changing the mass loss and wind velocity evolution into simple step functions (where the integrals are preserved) does not notably affect the resulting shell structure. The reason is that the formation of a thin shell happens very much through wind interaction, and since we do not change the wind velocity of the pre-flash phase or at the mass loss maximum, the wind interaction takes place in a similar fashion.

We return now to the importance of having a correct wind model as the basis for the boundary variations in the CSE model. In SS2000 the inner boundary of the atmosphere model is prescribed such that a mass loss rate is assumed and the computations start beyond the condensation radius where the wind velocity is set to some initial value (see Steffen et al. 1998, for details). The velocity field is thus not derived from a fully selfconsistent description of the stellar wind formation (although the equation of motion is solved, subject to the given boundary conditions). Moreover, the mass loss rate is not computed, but rather chosen according to a semi-empirical mass loss formula (Blöcker 1995). For the CSE evolution to be correcly modelled, however, we must be certain that the variations of both the mass loss rate and the wind velocity are essentially correct and consistent, since the interaction of wind phases is perhaps the most important ingredient.

Our detailed wind models (for $\Delta v_{\mathrm{p}}=4.0 \mathrm{~km} \mathrm{~s}^{-1}$ ) show that the wind velocity evolves from a slow phase $\left(\sim 10 \mathrm{~km} \mathrm{~s}^{-1}\right)$ before the He-shell flash, into a rather fast wind $\left(\sim 20 \mathrm{~km} \mathrm{~s}^{-1}\right)$ during He flash event and then back to a slower wind phase again (see Fig. 2). This is largely the same type of evolution used in the "modified He-shell flash scenario" in SS2000, although we have obtained it using a more sophisticated model, which provides both mass lass rate and wind velocity as functions of stellar parameters.

In the $\Delta v_{\mathrm{p}}=6.0 \mathrm{~km} \mathrm{~s}^{-1}$ case we obtain a very different evolution compared with the $\Delta v_{\mathrm{p}}=4.0 \mathrm{~km} \mathrm{~s}^{-1}$ case, also qualitatively speaking. The pre-flash mass loss rate is almost an order of magnitude higher and the wind velocity is a factor of two higher, providing almost no variations in the wind velocity 
before and during the flash. The latter is the main reason why the $\Delta v_{\mathrm{p}}=6.0 \mathrm{~km} \mathrm{~s}^{-1}$ case does not render a shell structure.

How reliable are these results then? One may ask whether, in some part, the pre-flash wind could be different if drift/coupling between the gas and dust phases was to be introduced. The question is very relevant since these effects are treated in SS2000. At the flash peak this is not a problem, since high mass loss rate results in a higher CSE density which makes the effects of drift much less significant. We are likely to overestimate the preflash wind velocity due to the phase coupling. Hence, the preflash wind velocity would probably be even lower if drift was to be included, making the velocity jump (see Fig. 3) even larger. Therefore, the inclusion of drift would probably not change the result qualitatively.

The thickness $\Delta R_{\mathrm{s}}$ of the shell is hard to evaluate in Case I, since the density peak has almost no spatial extension compared with the scales considered here, and the bump is probably a somewhat artificial structure created by a dramatic pressure increase at the shock fronts since no energy is radiated away. It is therefore difficult to interpret the meaning of the thickness as defined by the FWHM. In Case II the characteristic absolute thickness of the shell increases almost linearly with the shell radius (which in turn grows close to linearly with time). This results in a constant $\Delta R_{\mathrm{S}} / R_{\mathrm{s}}$-ratio, in agreement with the result found by SS2000 in the "modified He-shell flash scenario". However, where SS2000 find a ratio $\Delta R_{\mathrm{S}} / R_{\mathrm{S}} \approx 0.04$, we find this number to be roughly 0.01 , as we have pointed out previously in Sect. 3.2. If the faster wind associated with the "mass loss eruption" runs into a less dense medium, as in our model, it means that less matter is swept up and a thinner shell may thus be formed out of this interaction. But as far as we can tell, the thinner shells can not be explained only by the weaker pre-flash wind that we obtain, nor can it be explain by any significant difference in the duration of the mass loss eruption. A possible, additional explanation might be the fact that the shocks are isothermal with their surroundings in our model, and therefore the shell structure is not substantially broadened by thermal pressure. But the conditions in the SS2000 model are also close to isothermal, which makes this explanation seem less likely. A perhaps more likely explanation arises from the fact that the wind velocity jump from pre-flash to the flash-peak is an important parameter governing the formation of a detached shell. In the present work this jump is roughly a factor of 2 larger than in SS2000, which may be a viable explanation. In general, however, our results confirm the connection between stellar evolution (the He-shell flash) and the formation of detached geometrically thin shells around TP-AGB stars.

\section{Summary and conclusions}

We have used the RHD code for dynamic atmospheres by Höfner et al. (2003) in combination with a stellar evolutionary track of a $M=2 M_{\odot}, Z=0.01$ star in order to calculate the evolution of mass loss and wind velocity during a He-shell flash. The observed wind and mass loss properties associated with detached shells are rather well reproduced by our models of TP-AGB star atmospheres using stellar parameters sampled from this evolutionary track. None of the other existing mass loss prescriptions for AGB stars we have considered give, quantitatively speaking, mass loss rates similar to those which we compute. However, constraints on the mass loss evolution derived from observations (Olofsson et al. 2000; Schöier et al. 2005) appear to agree better with our results than with these mass loss formulae. Our numerical model may therefore be considered as a reasonably realistic description of the connection between stellar parameters and mass loss rates for TP-AGB stars.

We find that both mass loss rate and wind velocity increase during the flash event. This is in qualitative agreement with the "modified He-shell flash scenario" proposed in SS2000 and we thus conclude that geometrically thin detached shells are neither likely to be formed by simple two-wind interaction nor out of a "mass loss eruption" (with no variations in the wind velocity) alone. In fact, the formation of a slow and a fast wind combined with an eruptive mass loss associated with the fast wind turns out to be very critical. To use consistent combinations of mass loss rates and wind velocities is therefore most important in order to actually investigate a connection between He-shell flashes and detached shells. The amplitude of the internal pulsations of the star has a great effect on the wind velocities before and after the flash and thus on the strength of the wind interaction, in the case studied here.

Our simplified dynamical model of CSE evolution agrees qualitatively with what is expected from observations. We considered two basically complementary approximations for radiation effects. First we considered the case with no radiation effects at all (Case I) and then a second case where we used a simple analytic model for the temperature structure based on radiative equilibrium for the dust (Case II). In both cases we have assumed that all forces, except thermal pressure, are negligible. Case I and Case II agree qualitatively with each other, in the sense that they both produce a geometrically thin detached shell. However, only in Case II the relative thickness of the shell seems marginally consistent with $\mathrm{CO}$ observations. The thin shell structure seems to be well maintained throughout the evolutionary time span of the model, but the thickness of the shell grows linearely as it expands, which is most likely an effect of the internal pressure. Case I initially forms a shell structure with a much more pointed appearence, which in fact is the contact discontinuity at the interface between the winds. The pointed structure seems stable, but as the expansion continues a reverse shock propagating inwards is created, as well as an outgoing shock front which sweeps up a large portion of the matter ejected by the pre-flash wind. As a consequence of this, the shell structure evolves into a wide "density bump" with an unreasonably thin shell structure on top of it. From this we conclude that the temperature structure is one of the key components for the shell formation. In addition, simple 1D models may be inadequate since a full 3D simulation may result in much more complex structures that, in turn, may have significant effects on the formation and evolution of the shell.

Further work is obviously needed, but so far we can conclude that the He-shell flash and the associated "mass loss eruption" in combination with wind interaction gives a satisfying and consistent description of the formation of detached shells around TPAGB stars. Our detailed modelling of the mass loss evolution shows that a very short, intense period of mass loss can be associated with a He-shell flash. The "mass loss eruption" is followed by a period of no or very little mass loss, before a steady wind phase is once again established. This "eruptive" mass loss evolution does not seem to lend much support for the simple two-wind interaction scenario where a constant faster wind, with a higher mass loss rate associated with it, runs into a slower wind. Since a combination of a "mass loss eruption" and interacting wind phases is consistent with both stellar evolution and the observed properties of detached shells, we find this scenario to be the most likely.

This paper has presented a first attempt to construct a selfconsistent model of the formation of detached shells. It is, however, important to consider the CSE evolution in more detail than 
we have done here. A full RHD treatment in 3D would probably show additional features (e.g. instabilities) that might help to constrain the mass loss history in more detail. Our intention is to return to this issue in a future publication.

Acknowledgements. The authors wish to thank the referee, M. Steffen, for his careful reading of the manuscript and constructive criticism that helped to improve the clarity of this paper. B. Gustafsson is thanked for his reading of the manuscript draft and many valuable comments on the project. K. Eriksson and $\mathrm{R}$. Wahlin are thanked for stimulating discussions on AGB stars in general. This work was partly supported by the Swedish Research Council (Vetenskapsrådet). F.H. acknowledges support by the LDRD program (20060357ER) at Los Alamos National Laboratory. Part of this work has been carried out in collaboration with the NSF Physics Frontier Center JINA (Joint Insititute for Nuclear Astrophysics).

\section{References}

Andersen, A. C., Höfner, S., \& Gautschy-Liodl, R. 2003, A\&A, 400, 981 Bowen, G. H. 1988, ApJ, 329, 299

Blöcker, T. 1995, A\&A, 297, 727

Dorfi, E., \& Drury, L. O'C. 1987, Comput. Phys. Commun., 69, 175

Dorfi, E., \& Feuchtinger, M. 1995, Comput. Phys. Commun., 89, 69

Feast, M. W., Glass, I. S., Whitelock, P. A., \& Catchpole, R. M. 1989, MNRAS, 241,375

Gail, H.-P., \& Sedlmayr, E. 1988, A\&A, 206, 153

Gauger, A., Gail, H.-P., \& Sedlmayr, E. 1990, A\&A, 235, 345

Gautschy-Loidl, R., Höfner, S., Jorgensen, U. G., \& Hron, J. 2004, A\&A, 422, 289

González-Delgado, D., Olofsson, H., Schwarz, H. E., Eriksson, K., \& Gustafsson, B. 2001, A\&A, 372, 885

Herwig, F. 2000, A\&A, 360, 952

Herwig, F. 2004, ApJ, 605, 425
Herwig, F., Austin, S., \& Lattanzio, J. 2006, PRC, 73:2, 25802

Höfner, S., \& Dorfi, E. A. 1997, A\&A, 319, 648

Höfner, S., Feuchtinger, M. U., \& Dorfi, E. A. 1995, A\&A, 279, 815

Höfner, S., Gautschy-Loidl, R., Aringer, B., \& Jørgensen, U. G. 2003, A\&A, 399,589

Lamers, H. J. G. L. M., \& Cassinelli, J. P. 1999, Introduction to Stellar Winds (Cambridge University Press)

Kwok, S., Purton, C. R., \& FitzGerald, P. M. 1978, ApJ, 219, 125

Myasnikov, A. V., Belov, N. A., Gustafsson, B., \& Eriksson, K. 2000, Ap\&SS, 274,231

Nowotny, W., Aringer, B., Höfner, S., Gautschy-Loidl, R., \& Windsteig, W. 2005a, A\&A, 437, 273

Nowotny, W., Lebzelter, T., Hron, J., \& Höfner, S. 2005b, A\&A, 437, 285

Olofsson, H., Eriksson, K., \& Gustafsson, B. 1987, A\&A, 183, L13

Olofsson, H., Carlstrom, U., Eriksson, K., Gustafsson, B., \& Willson, L.-A. 1990, A\&A, 230, L13

Olofsson, H., Eriksson, K., Gustafsson, B., \& Carlström, U. 1993, ApJS, 87, 267

Olofsson, H., Bergman, P., Lucas, R., et al. 1998, A\&A, 330, L1

Olofsson, H., Bergman, P., Lucas, R., et al. 2000, A\&A, 353, 583

Reimers, D. 1975, Mem. Soc. Roy. Sci. Liège, 6e Ser., 8, 369

Rouleau, F., \& Martin, P. G. 1991, ApJ, 377, 526

Sandin, C., \& Höfner, S. 2003, A\&A, 404, 789

Sandin, C., \& Höfner, S. 2004, A\&A, 413, 789

Schöier, F. L., \& Olofsson, H. 2001, A\&A, 368, 969

Schöier, F. L., Lindqvist, M., \& Olofsson, H. 2005, A\&A, 436, 633

Steffen, M., \& Schönberner, D. 2000, A\&A, 357, 180 (SS2000)

Steffen, M., Szczerba, M. R., Men'shchikov, A., \& Schönberner, D. 1997, A\&A, 126,39

Steffen, M., Szczerba, M. R., \& Schönberner, D. 1998, A\&A, 337, 149

Vassiliadis, E., \& Wood, P. R. 1993, ApJ, 413, 614

van Leer, B. 1977, J. Comput. Phys., 23, 276

Wachter, A., Schröder, K.-P., Winters, J. M., Arndt, T. U., \& Sedlmayr, E. 2002, A\&A, 384, 452

Wareing, C. J., Zijlstra, A. A., Speck, A. K., et al. 2006, MNRAS, 372, L63

Zuckerman, B. 1993, A\&A, 276, 367 
L. Mattsson et al.: Mass loss evolution and the formation of detached shells around TP-AGB stars, Online Material p 1

\section{Online Material}


L. Mattsson et al.: Mass loss evolution and the formation of detached shells around TP-AGB stars, Online Material p 2

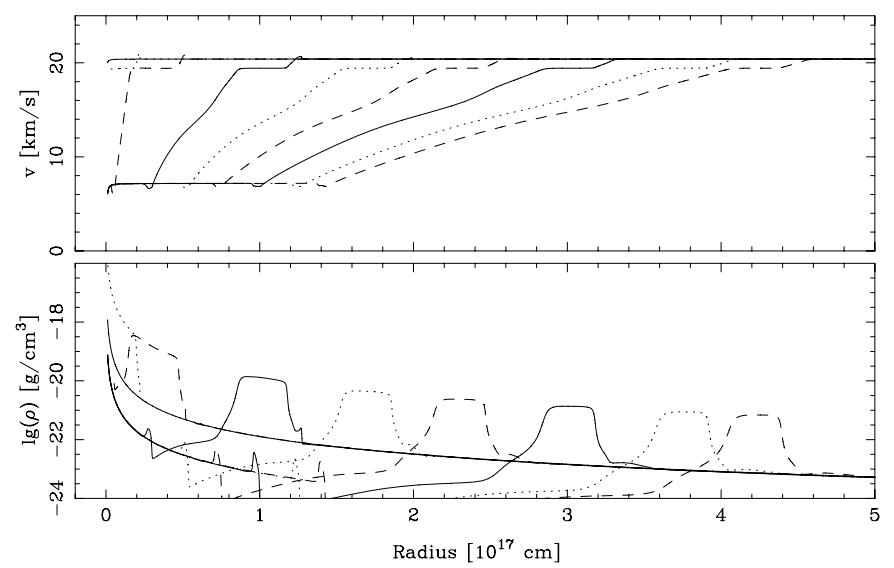

Fig. A.1. Nine instants in the evolution of the CSE. Upper panel: velocity as function of radius for Case I with the same boundary condition as "case B" in SS2000. Lower panel: density as function of radius for Case I with the same boundary condition as "case B" in SS2000.

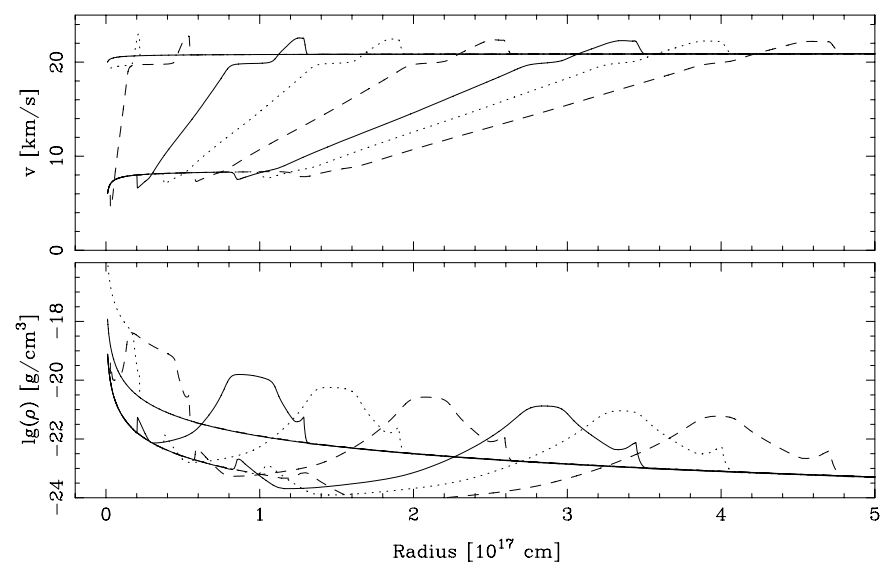

Fig. A.2. Same as Fig. A.1, but for Case II.

\section{Appendix A: Comparison with case " $B$ " and " $C$ " in SS2000}

This appendix presents test cases using basically the same boundary variations as in SS2000. Their "NEBEL" code contains a detailed treatment of radiation and cooling, compared with our fixed temperature structure in Case II. The "DEXCEL" runs have no directly corresponding case among the ones we have tried here. But since the "DEXCEL" code is known to suffer from numerical diffusion (which was pointed out in SS2000) any direct comparison would be difficult. Nonetheless, we think it is important to show that our code (Case II) produces results that are comparable to the "NEBEL" runs presented in SS2000 if the input is similar.

Using a simple mass loss evolution of the kind used in SS2000 (i.e. step functions, as in Sect. 3.2) in our Case II, which is quite similar to the "NEBEL" models of SS2000, we are able to basically reproduce their results, i.e. the wind velocity field and the mass density of the CSE (Figs. A.2 and A.4) evolve in ways that are very similar to their "case B" and "case C". In Case I we obtain a result that shows some resemblance to the result from the "DEXCEL" model of SS2000, but we see no obvious signs of numerical diffusion. The corresponding plots for Case I (Figs. A.1 and A.3) are shown for comparison.

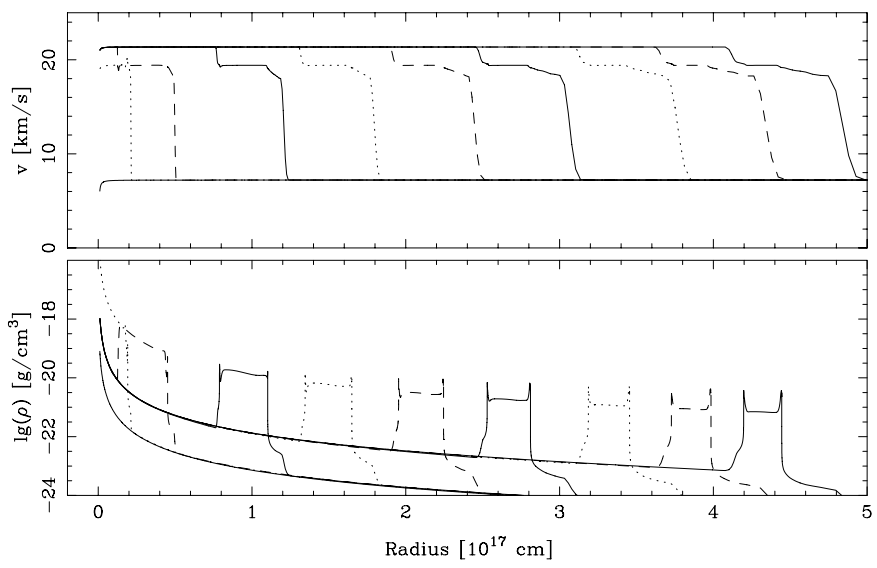

Fig. A.3. Nine instants in the evolution of the CSE. Upper panel: velocity as function of radius for Case I with the same boundary condition as "case C" in SS2000. Lower panel: density as function of radius for Case I with the same boundary condition as "case C" in SS2000.

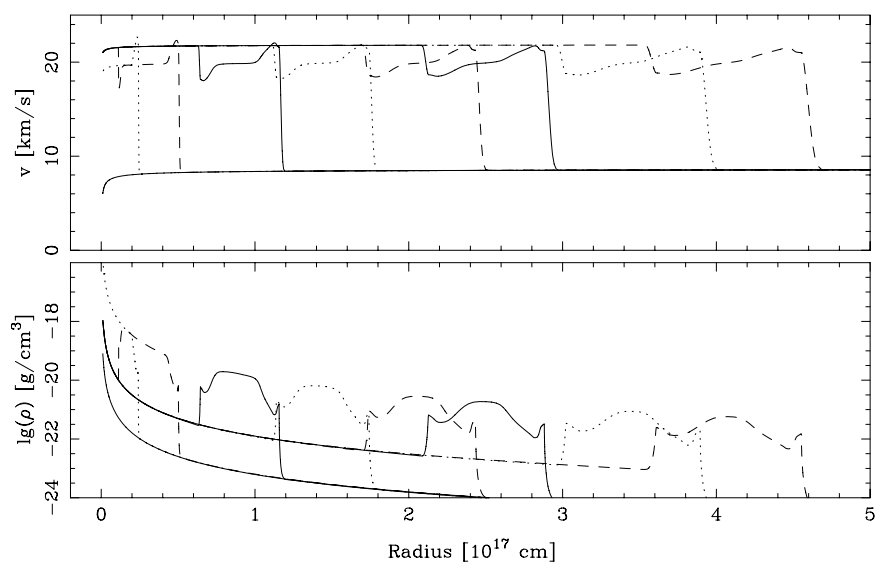

Fig. A.4. Same as Fig. A.3, but for Case II. 